\title{
Nutrient-dependent control of RNA polymerase II elongation rate regulates specific gene expression programs by alternative polyadenylation
}

\author{
Carlo Yague-Sanz, ${ }^{1,6}$ Yann Vanrobaeys, ${ }^{1,5,6}$ Ronan Fernandez, ${ }^{2,3}$ Maxime Duval, ${ }^{1}$ Marc Larochelle, ${ }^{1}$ \\ Jude Beaudoin,, Julien Berro, ${ }^{2,3}$ Simon Labbé, ${ }^{1}$ Pierre-Étienne Jacques, ${ }^{4}$ and François Bachand ${ }^{1}$ \\ ${ }^{1}$ Department of Biochemistry and Functional Genomics, Université de Sherbrooke, Sherbrooke, Québec J1E 4K8, Canada; \\ ${ }^{2}$ Department of Molecular Biophysics and Biochemistry, ${ }^{3}$ Department of Cell Biology, Yale School of Medicine, New Haven, \\ Connecticut 06520, USA; ${ }^{4}$ Department of Biology, Université de Sherbrooke, Sherbrooke, Québec J1K 2R1, Canada
}

Transcription by RNA polymerase II (RNAPII) is a dynamic process with frequent variations in the elongation rate. However, the physiological relevance of variations in RNAPII elongation kinetics has remained unclear. Here we show in yeast that a RNAPII mutant that reduces the transcription elongation rate causes widespread changes in alternative polyadenylation (APA). We unveil two mechanisms by which APA affects gene expression in the slow mutant: 3' UTR shortening and gene derepression by premature transcription termination of upstream interfering noncoding RNAs. Strikingly, the genes affected by these mechanisms are enriched for functions involved in phosphate uptake and purine synthesis, processes essential for maintenance of the intracellular nucleotide pool. As nucleotide concentration regulates transcription elongation, our findings argue that RNAPII is a sensor of nucleotide availability and that genes important for nucleotide pool maintenance have adopted regulatory mechanisms responsive to reduced rates of transcription elongation.

[Keywords: transcription elongation rate; alternative polyadenylation; RNA polymerase II; phosphate starvation; transcription termination; NTP sensing]

Supplemental material is available for this article.

Received January 28, 2020; revised version accepted May 6, 2020.

Transcription of eukaryotic genes by RNA polymerase II (RNAPII) is a fundamental process composed of three sequential, yet interconnected steps that consist of initiation, elongation, and termination. During this transcription cycle, transcription units are generally characterized by a conserved 5' $5^{\prime}$-to-3' profile of phosphorylation on the carboxy-terminal domain (CTD) of Rpb1, the catalytic subunit of RNAPII. Rpb1 CTD heptad repeats, which consist of the consensus amino acid sequence $\mathrm{Y}_{1}-\mathrm{S}_{2}-\mathrm{P}_{3}-\mathrm{T}_{4}-\mathrm{S}_{5}$ $\mathrm{P}_{6}-\mathrm{S}_{7}$, undergo dynamic changes in phosphorylation during transcription elongation, such that Ser5 phosphorylation (Ser5P) typically peaks at the $5^{\prime}$ end, whereas Ser2P is highest at the $3^{\prime}$ end (Zaborowska et al. 2016; Harlen and Churchman 2017). These CTD phosphorylation and dephosphorylation cycles during the elongation phase are key to coordinate the sequential recruitment of RNA

${ }^{5}$ Present address: Interdisciplinary Graduate Program in Genetics, University of Iowa, Iowa City, IA 52242, USA.

${ }^{6}$ These authors contributed equally to this work.

Corresponding author: f.bachand@usherbrooke.ca

Article published online ahead of print. Article and publication date are online at http://www.genesdev.org/cgi/doi/10.1101/gad.337212.120. Freely available online through the Genes \& Development Open Access option. processing factors along transcription units. For instance, at the $3^{\prime}$ end of genes, Ser2P contributes to the cotranscriptional recruitment of conserved $3^{\prime}$ end processing factors such as Pcf11 and Seb1, which, together with cleavage and polyadenylation factors, promote endonucleolytic RNA cleavage and polyadenylation [poly(A)] site selection (Meinhart and Cramer 2004; Lunde et al. 2010; Lemay et al. 2016; Wittmann et al. 2017). In addition to releasing the nascent transcript for polyadenylation, the cleavage mediated by the $3^{\prime}$ end processing machinery also provides an uncapped $5^{\prime}$ entry point for a conserved protein complex that includes the $5^{\prime}-3^{\prime}$ exonuclease Rat1/Xrn2 (Kim et al. 2004; West et al. 2004). This complex is thought to promote transcription termination by chasing RNAPII and ultimately triggering RNAPII dissociation along the chromatin template (Porrua et al. 2016; Proudfoot 2016). Termination of RNAPII transcription thus requires cleavage at the poly(A) site followed by cotranscriptional degradation of the downstream nascent transcript.

(C) 2020 Yague-Sanz et al. This article, published in Genes \& Development, is available under a Creative Commons License (Attribution $4.0 \mathrm{In}$ ternational), as described at http://creativecommons.org/licenses/by/4.0/. 
Transcription elongation is normally viewed as a continuous process during which RNAPII traverses the coding region after initiating at a promoter. However, genome-wide studies indicate that transcription elongation by RNAPII is a rather intermittent process, with frequent occurrences of pausing, backtracking, and arrest (Churchman and Weissman 2011; Gómez-Herreros et al. 2012; Sheridan et al. 2019). Furthermore, rates of transcription elongation in coding regions appear to differ between genes and within transcriptional units (Danko et al. 2013; Jonkers et al. 2014; Veloso et al. 2014; Cortazar et al. 2019). As several key steps of RNA maturation occur cotranscriptionally, variations in elongation rates are therefore expected to have important consequences on the efficiency of pre-mRNA processing. For example, using Rpb1 catalytic mutants that transcribe faster or slower than normal, it has been shown that changes in the transcription elongation rate can affect the outcome of premRNA splicing in both yeast and human cells (Roberts et al. 1998; de la Mata et al. 2003; Fong et al. 2014; Aslanzadeh et al. 2018). Conversely, pre-mRNA splicing also appears to affect transcription elongation rates (Kornblihtt et al. 2004; Alexander et al. 2010), suggesting that both processes mutually contribute to promote efficient and accurate coupling between transcription and RNA processing. Current models therefore suggest that regulation of RNAPII elongation kinetics can influence splicing such that slowing down the rate of transcription elongation increases the opportunity of an upstream splice site to be recognized before a competing downstream splice site is transcribed (Bentley 2014).

Another cotranscriptional process potentially controlled by RNAPII elongation rate is poly(A) site selection in alternative polyadenylation (APA). APA occurs when genes harbor multiple poly(A) sites, which give rise to mRNA isoforms with $3^{\prime}$ untranslated regions (UTRs) of different lengths (Tian and Manley 2017). Accordingly, it has been proposed that faster elongation rates would increase the competition between proximal and distal poly (A) sites, whereas a slow transcribing RNAPII would favor usage of proximal poly(A) sites (Pinto et al. 2011; Liu et al. 2017a). APA plays important roles in posttranscriptional gene regulation, as $3^{\prime}$ UTR elements contain sequence motifs that can regulate gene expression via RNA stability, translation control, and RNA localization (Mayr 2017).

Although evidence strongly supports the view that the rate of RNAPII elongation can influence cotranscriptional RNA processing, how kinetics of RNAPII passage through chromatin globally affects gene expression remains poorly understood. Furthermore, whether variations in transcription elongation rate are used in a natural context to control the expression of a specific set of genes is largely unknown. In this work, we analyzed the genome-wide expression changes imposed by a Rpbl catalytic mutant with a reduced elongation rate in fission yeast. We found that a conserved core regulon sensitive to phosphate starvation (PHO regulon) is activated by a slowly transcribing RNAPII via a mechanism that involves APA and premature transcription termination of interfering upstream long noncoding (lnc) RNAs. Importantly, we show that APA and premature termination are naturally used to regulate this core PHO regulon upon phosphate starvation. Collectively, our data reveal that a reduction in the transcription elongation rate causes a distal-to-proximal shift in APA, resulting in increased expression of a specific set of genes that includes the core $\mathrm{PHO}$ regulon and genes involved in the purine biosynthesis pathway. Our findings support a model in which phosphate limitation results in reduced elongation rates as a consequence of nucleotide depletion and that phosphate-responsive genes have evolved activation mechanisms sensitive to suboptimal transcription elongation kinetics.

\section{Results}

A mutant of RNA polymerase II with a reduced elongation rate causes specific changes in gene expression

To investigate how a slow transcription elongation rate globally affects gene expression, we introduced a mutation that was originally identified in a genetic screen for hypersensitivity to the nucleotide-depleting drug 6-azauracil (6AU) in S. cerevisiae (Malagon et al. 2006) in the gene encoding $S$. pombe $r p b 1$, the largest subunit of RNA polymerase II (RNAPII). This mutation substitutes an evolutionarily conserved asparagine at position 494 of $S$. pombe Rpb1 to an aspartic acid residue (N494D) (see Fig. 1A) and corresponds to the N488D substitution in $S$. cerevisiae Rpb1 that results in reduced elongation rates in vitro (Malagon et al. 2006) and in vivo (JimenoGonzález et al. 2010) as well as in increased transcriptional slippage (Strathern et al. 2013). Asparagine 494 is located below the active site in the Rpb1 catalytic loop (see Supplemental Fig. S1), in the vicinity of the magnesium ions and the incoming nucleoside triphosphates (Strathern et al. 2013). To generate a mutant strain that expresses Rpb1 N494D from the endogenous $r p b 1$ locus, we used CRISPR/Cas9-based genome editing (Fernandez and Berro 2016) and isolated independent clones that displayed the desired $r p b 1$ mutation after DNA sequencing. Importantly, the rpb1-N494D mutant was sensitive to 6AU and mycophenolic acid (MPA) (Supplemental Fig. S2), drugs known to deplete intracellular NTP pools and stress transcription elongation, as previously shown for the analogous $S$. cerevisiae mutant (Malagon et al. 2006).

To measure the impact of the RNAPII slow mutant on gene expression, we analyzed total RNA prepared from two independent $r p b 1-N 494 D$ clones as well as the parental control strain by strand-specific RNA-seq. Expression data from the two N494D mutants clustered together (Supplemental Fig. S3A), showing strong reproducibility between the independent clones. In total, 323 genes were down-regulated and 457 were up-regulated in the slow mutant (Fig. 1B; Supplemental Table S1). To further validate our RNA-seq experiment, we measured the change of expression of 10 differentially expressed genes by RTqPCR and observed good overall correlation $\left(R^{2}=0.92\right)$ with the RNA-seq data (Supplemental Fig. S3B,C). Down-regulated genes were enriched for gene ontology 


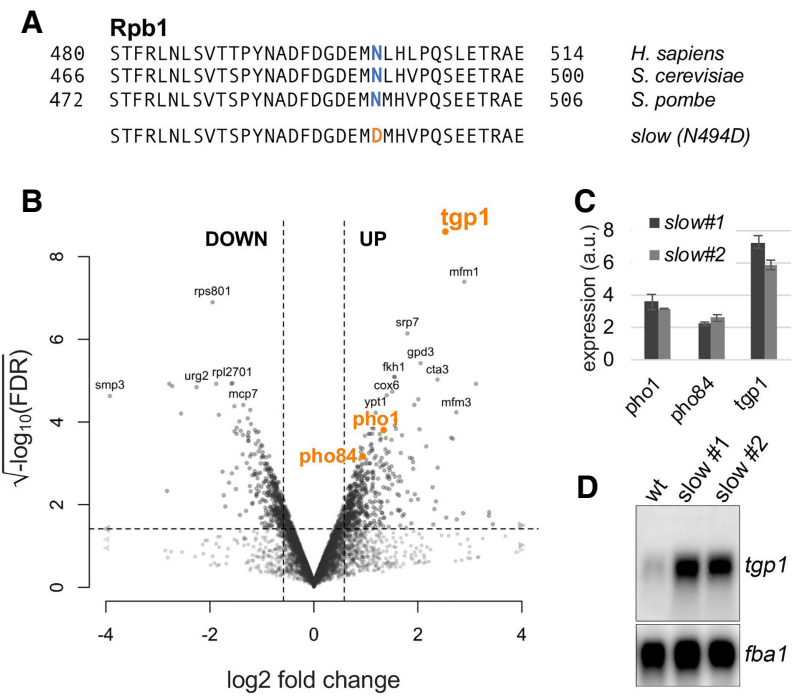

Figure 1. Transcriptome-wide analysis of gene expression changes in the $S$. pombe Rpbl slow mutant. (A) Multiple sequence alignment of a conserved region in the catalytic core of Rpb1. The conserved asparagine residue is colored in blue, whereas the substitution is colored in orange in the slow mutant. $(B)$ Volcano plot of statistical significance against fold change (in $\log _{2}$ ) of gene expression in the slow mutant relative to the wildtype control ( $n=7049$ genes). The dashed lines represent the thresholds for calling significant differential gene expression: FDR $<0.01$ and absolute $\log _{2}$ fold change $>\log _{2}(1.5)$. To ease viewing, genes with values beyond axes limits are represented by arrowheads. The phosphate-responsive genes tgp1, pho1, and pho84 are highlighted in orange. (C) RT-qPCR analysis of $\operatorname{tgp} 1$, pho1, and pho84 mRNA levels in independent slow mutants relative to the wild-type parental strain from three independent experiments. (a.u.) Arbitrary units. Error bars represent the standard deviation of the mean. $(D)$ Validation of tgp1 up-regulation in independent Rpb1 slow mutants by Northern blot. The fba1 mRNA was used as a loading control.

categories related to transmembrane transport, ribosome biogenesis, and iron assimilation (Supplemental Fig. S4A), whereas up-regulated genes were significantly enriched for genes involved in purine (especially ATP) biosynthesis as well as sexual reproduction (Supplemental Fig. S4B). Many of the up-regulated genes in the slow mutant are direct targets of Ste11, which is induced in the slow mutant and functions as the master transcription factor for sexual development in fission yeast. Thus, upregulation of genes such as mei2, $m f m 1, m f m 2$, and $\mathrm{mfm} 3$ is likely to be an indirect consequence of ste11 induction.

We also examined for splicing deficiencies in the $r p b 1$ slow mutant, as previous studies indicate that RNAPII elongation rate can influence splicing efficiency (de la Mata et al. 2003; Fong et al. 2014; Aslanzadeh et al. 2018). Two independent computational approaches were used on our RNA-seq data to examine the impact of RNAPII slowdown on splicing efficiency (Li et al. 2020). However, none of these analyses supported strong global changes in RNA splicing in the rpb1 slow mutant (Supplemental
Fig. S5). Moreover, intron-containing genes were neither enriched in the set of genes that were significantly up-regulated nor in the set of genes significantly down-regulated in the slow mutant. We therefore conclude that the rpb1 N494D slow mutant affects the expression of a specific set of genes but does not largely influence RNA splicing.

\section{A conserved regulon that responds to phosphate starvation is induced in the RNAPII slow mutant}

Fission and budding yeasts share a core regulon responsible for the initial response to phosphate limitations comprising the pho1, pho84, and tgp1 genes, which encode phosphate harvesting and transport proteins (CarterO'Connell et al. 2012). Notably, all three genes of the core $\mathrm{PHO}$ regulon were significantly up-regulated in the RNAPII slow mutant (Fig. 1B); yet, expression from those genes should have been repressed as cells were grown in phosphate-containing media. These results were validated by RT-qPCR for the pho1, pho84, and tgp1 genes (Fig. 1C) as well as by Northern blot analysis for the $\operatorname{tgp} 1$ mRNA (Fig. 1D). These data reveal that expression of the conserved core PHO regulon is particularly sensitive to a reduction in the transcription elongation rate.

Premature termination of nc-tgp $1 \operatorname{lncRNA}$ transcription allows for tgp 1 induction in the RNAPII slow mutant

To begin to elucidate the molecular mechanisms by which the slow RNAPII mutant results in gene activation, we focused on $\operatorname{tgp} 1$, which is the most highly induced gene in the Rpbl slow mutant based on the RNA-seq data. $\operatorname{tgp} 1$ expression has been previously studied in fission yeast (Ard et al. 2014; Ard and Allshire 2016; Sanchez et al. 2018a) and shown to be negatively regulated by a long noncoding (lnc) RNA expressed from the upstream nc-tgp1 locus (Fig. 2A). However, the exact mechanism by which the repressive effect of $n c$ - $\operatorname{tgp} 1$ transcription is disabled to allow $\operatorname{tgp} 1$ activation upon phosphate starvation has been elusive. Interestingly, our transcriptomewide analysis of poly(A) site (PAS) mapping in $S$. pombe using 3'READS (Liu et al. 2017b) indicated the presence of at least two PASs in the nc-tgp1 locus: a minor proximal PAS and a major distal PAS, the latter being surrounded by strong consensus motifs (Figs. 2A,B, 3D). Notably, by comparing the RNA-seq coverage in $n c-\operatorname{tg} p 1$ and $\operatorname{tgp} 1$ between the wild-type strain and the slow mutant, we found that whereas $\operatorname{tgp} 1 \mathrm{mRNA}$ levels were upregulated in the RNAPII slow mutant (Fig. 2B, see red over blue signal), the levels of the upstream nc-tgp1 lncRNA were strongly repressed in the slow mutant (Fig. 2B, blue over red signal). This observation was validated by RT-qPCR analysis using primer pairs positioned along the nc-tgp1 and $\operatorname{tgp} 1$ loci (see Fig. 2A), confirming the reduction of $n c-\operatorname{tg} p 1$ lncRNA concomitant with increased tgp1 mRNA levels in the slow mutant (Fig. 2C). Similar observations were seen for the other two core PHO regulon genes, pho84 and pho1 (Supplemental Fig. S6A). Reduction of the nctgp1 lncRNA in the RNAPII slow mutant was also confirmed by RNase protection assays (Supplemental Fig. 


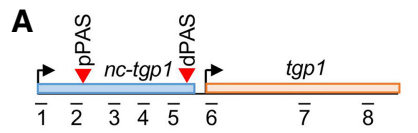

C

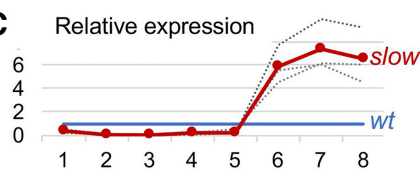

D
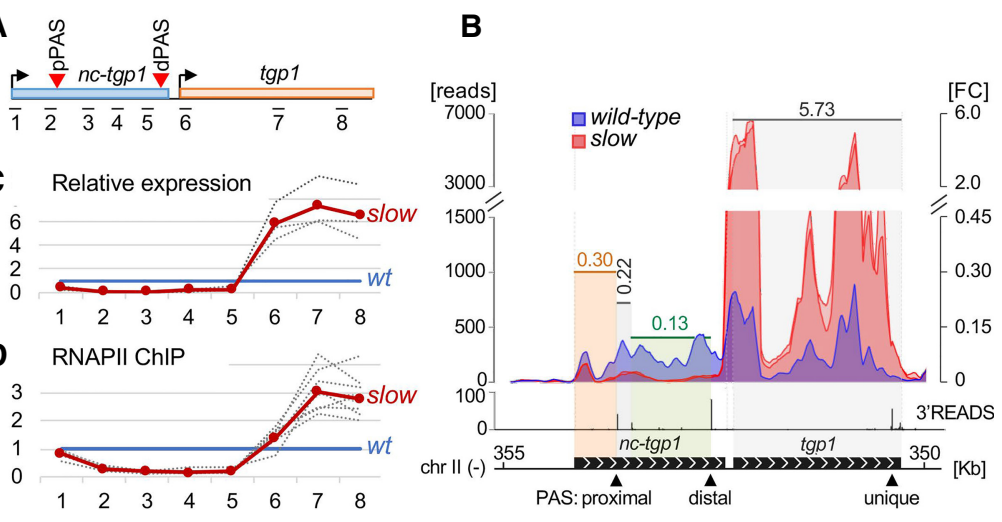

nc-tgp1/tgp1 expression relative to wild-type cells in three independent experiments. The blue and red lines correspond to the baseline wild-type level and the mean value for the slow mutant, respectively. The individual replicates are shown as dashed lines. $(D)$ Relative RNAPII occupancy in the slow mutant compared with the wild type at the nc-tgp1/tgp1 locus from nine independent experiments. The blue and red lines correspond to the baseline wild-type level and the mean value, respectively, for the slow mutant. The individual replicates are shown as dashed lines.

S6B). Importantly, by segmenting the nc-tgp1 locus into different regions based on PAS identification (Liu et al. 2017b), we noted a 5'-to-3' decrease in the RNA-seq signal ratio in the mutant relative to the wild-type strain (Fig. $2 \mathrm{~B})$, suggesting a reduction in the production of the fulllength $n c$-tgp1 lncRNA. One mechanism by which the RNAPII slow mutant could result in decreased production of full-length $n c$-tgp1 is by increased usage of the proximal PAS. As transcription termination is intimately coupled to RNA 3' end processing (Porrua et al. 2016; Proudfoot 2016), this would be expected to trigger premature termination. To test this hypothesis, we analyzed RNAPII occupancy by chromatin immunoprecipitation (ChIP) assays. As shown in Figure 2D, the Rpb1 slow mutant demonstrated increased RNAPII density in the $\operatorname{tgp} 1$ locus relative to the control strain (see regions 6-8), consistent with up-regulation of $\operatorname{tgp} 1$ mRNA in $r p b 1-N 494 D$ cells. In contrast, RNAPII levels markedly declined over the proximal nc-tgp 1 poly(A) site in the slow mutant (regions 2-5), consistent with the idea that the slower elongation rate in rpb1-N494D cells promotes premature transcription termination at the nc-tgp1 locus. Our data argue that the induction of tgp1 expression in the RNAPII slow mutant results from premature termination of $n c$ $\operatorname{tgp} 1$ transcription.

Enrichment of $3^{\prime}$ end processing factors at the proximal nc-tgp1 PAS in the RNAPII slow mutant

ChIP-seq analyses of components of the RNA $3^{\prime}$ end processing machinery show enrichment at the $3^{\prime}$ end of genes (Larochelle et al. 2018) where they promote cotranscriptional RNA cleavage and transcription termination. To measure the effect of the RNAPII slow mutant on the recruitment of the $3^{\prime}$ end processing machinery at the $n c$ $\operatorname{tgp} 1$ locus, we used ChIP analysis to monitor the binding profile of Rna14, a conserved component of the cleavage and polyadenylation factor I complex (Casañal et al. 2017). To account for differences in transcription profiles
Figure 2. Premature termination of $n c-\operatorname{tgp} 1$ transcription in the RNAPII slow mutant. (A) On-scale representation of the $n c-\operatorname{tgp} 1 / \operatorname{tgp} 1$ locus indicating the position of the amplicons (1-8) used for ChIP and qRT-PCR analyses throughout this study as well as the position of the proximal (pPAS) and distal (dPAS) poly(A) sites in nc-tgp1. (B) Normalized read coverage (left axes) at the nc-tgp1/tgp1 locus for the wild-type (blue area) and slow mutants (red areas) based from the RNA-seq data, and for previously published 3' READS data (Liu et al. 2017b) in wildtype cells (black peaks at the bottom). The fold change (FC; right axis) is calculated between averaged mutant versus wild-type values for gene segments delimited by $3^{\prime}$ READS peaks, which are represented by shaded vertical areas. (C) RT-qPCR analysis of ts. The blue and red lines correspond to the baseline
ual replicates are shown as dashed lines. $(D)$ Relative observed between the slow mutant and the wild-type strain (Fig. 2D), we normalized Rna14 ChIP values to RNAPII occupancy by performing simultaneous measurements of Rnal4 and Rpb1 recruitment from the same chromatin preparations. Consistent with the idea that the RNAPII slow mutant favors premature termination of $n c-\operatorname{tg} p 1$ transcription, Rna14 was strongly enriched around the proximal PAS of nc-tgp1 in the slow mutant compared with the wild-type (Fig. 3A, see regions 2-3), whereas reduced Rna14 levels were detected near the distal poly(A) site of $n c-t g p 1$ (Fig. 3A, see regions 5-6). We also monitored the recruitment pattern of Seb1, a RNAPII-associated factor that is important for PAS selection and transcription termination of coding and noncoding genes (Lemay et al. 2016; Wittmann et al. 2017). Similar to Rna14, we observed a redistribution of Seb1 from the distal to the proximal PAS of $n c-\operatorname{tgp} 1$ (Fig. 3B). These results suggest that the slower elongation rate of the Rpb1 N494D mutant enhances the cotranscriptional recognition of suboptimal proximal PASs in $n c-\operatorname{tg} p 1$, leading to premature transcription termination.

RT-qPCR and ChIP assays were also used to assess whether APA-dependent premature termination of lncRNA transcription was responsible for derepression of the other core PHO regulon genes, namely, pho84 and pho1. Importantly, RT-qPCR analysis confirmed the derepression of pho84 and pho1 in the rpb1 mutant (amplicons 6 and 12-13, respectively) (Supplemental Fig. S6C,D), whereas the prt2 and nc-pho1 lncRNAs were both downregulated (amplicons 3-4 and 8-11, respectively). Similar to the mechanism of tgp1 derepression, RNAPII and Rna14 ChIP data support that the prt2 lncRNA undergoes premature transcription termination in the rpb1 slow mutant, leading to the activation of pho84 (Supplemental Fig. $\mathrm{S} 6 \mathrm{E}, \mathrm{F})$. However, it remains unclear whether such a mechanism also applies to the nc-pho1/pho1 locus. Indeed, the proximity between the $3^{\prime}$ end of the pho 84 gene and the $n c$ pho1 promoter makes the RNAPII and Rna14 ChIP data difficult to interpret (Supplemental Fig. S6E,F). One 

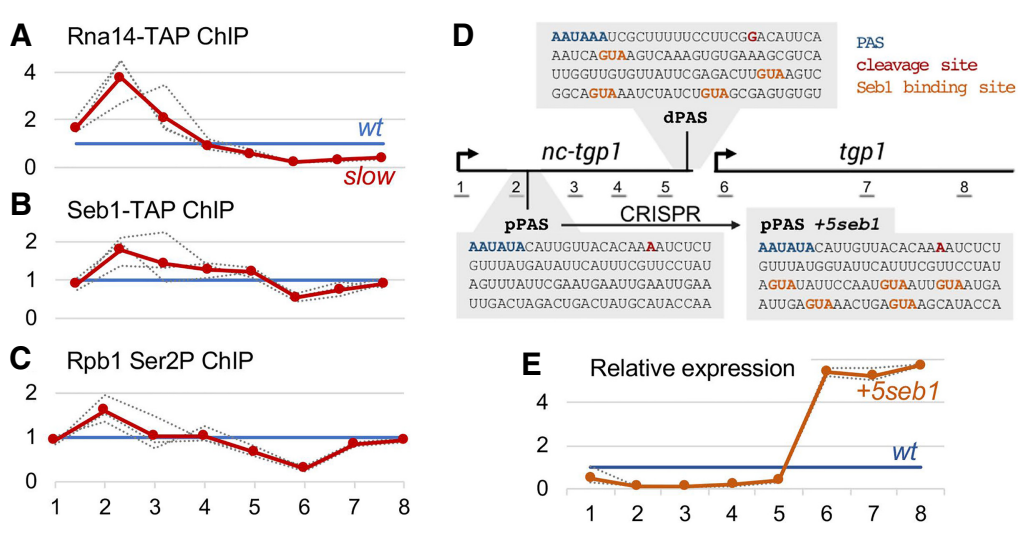

Figure 3. Redistribution of $3^{\prime}$ end processing factors and CTD Ser2 phosphorylation to the proximal poly (A) site of $n c$-tgp 1 in the RNAPII slow mutant. $(A-C)$ Chromatin occupancy of Rnal4 $(A)$, Seb1 $(B)$, and Rpb1 CTD Ser2 phosphorylation $(C)$ at the nc-tgp1/ $\operatorname{tgp} 1$ locus in the RNAPII slow mutant (red line) relative to the wild-type control (blue line). Individual replicates $(n=3$; dashed lines) were normalized to total RNAPII levels measured from the same chromatin preparation at each region and expressed relative to the wild type. The positions of the amplicons (1-8) used throughout this study are shown in Figure 3D. (D) On-scale representation of the $n c-\operatorname{tgp} 1 / \operatorname{tgp} 1$ locus indicating the nucleotide sequences surrounding the proximal and distal poly(A) sites (pPAS and dPAS, respectively) of nc-tgp1. The modifications introducing five Seb1-binding sites downstream from the proximal nc-tgp 1 (pPAS $+5 s e b 1$ mutant) by CRISPR are shown at the right. (E) RTqPCR analysis of $n c$-tgp1/tgp1 expression relative to wild type from three independent +5 seb1 clones. The blue and orange lines correspond to the baseline wild-type level and the mean value for the +5 seb1 mutant, respectively. The individual replicates are shown as dashed lines.

possibility is that the derepression of pho84 as a consequence of premature termination of the prt2 lncRNA might result in transcriptional interference at the $n c$ pho1 promoter, thereby repressing nc-pho1 transcription and ultimately coupling pho84 and pho1 derepression.

Next, we wondered which molecular feature of the Rpb1 N494D protein might lead to an increase in premature termination at the nc-tgp1 locus. Since CTD Ser2 phosphorylation (Ser2P) coincides with the recruitment of the $3^{\prime}$ end processing machinery (Ahn et al. 2004; Meinhart and Cramer 2004), we analyzed CTD Ser2P levels across the $n c$ - $\operatorname{tgp} 1$ gene in wild-type and $r p b 1-N 494 D$ cells. ChIP experiments were performed using an antibody specific for CTD Ser2P (Larochelle et al. 2018) and normalized to levels of total Rpb1 in the respective regions. Although total levels of Ser2 CTD phosphorylation were similar between the wild-type and the slow mutant (Supplemental Fig. S7A), decreased levels of CTD Ser2P were detected at the distal PAS (regions 5-6) of nc-tgp 1 together with a concomitant increase at the proximal PAS (Fig. 3C, see region 2). As Ser2P is strongly associated with $3^{\prime}$ end processing, increased levels of this CTD modification near the proximal PAS of $n c-\operatorname{tgp} 1$ is consistent with premature termination of $n c-\operatorname{tg} p 1$ transcription in the RNAPII slow mutant. We also examined the levels of Thr4 and Ser7 CTD phosphorylation at the nc-tgp1/ $\operatorname{tgp} 1$ locus, as antagonistic functions in transcription termination were reported for these CTD modifications in fission yeast (Sanchez et al. 2018b). Specifically, Ser7P and Thr4P were shown to have negative and positive effects, respectively, on transcription termination. Consistent with these findings, the RNAPII slow mutant showed decreased levels of Ser7P concurrent with increased Thr4P signal at the nc-tgp1 locus (Supplemental Fig. S7C,D) where we found increased levels of premature transcription termination in the slow mutant (Figs. 2, 3). The levels of Ser5 CTD phosphorylation across the $n c$ $\operatorname{tgp} 1$ locus showed a slight redistribution toward the distal PAS in rpb1-N494D cells (Supplemental Fig. S7E), which could be a consequence of $\operatorname{tgp} 1$ transcriptional activation.
To assess whether premature termination of $n c$-tgp1 transcription was sufficient to induce $\operatorname{tgp} 1$ expression, we designed a strategy to strengthen the proximal PAS of nc-tgp1. Upon examination of the sequences surrounding the two major nc-tgp1 poly(A) signals, we noticed that whereas consensus 5'-GUA-3' Seb1-binding motifs (Lemay et al. 2016) were found downstream from the distal PAS, there was no Seb1 motif downstream from the proximal PAS (Fig. 3D). Because Seb1 promotes RNAPII pausing, poly(A) site selection, and transcription termination (Lemay et al. 2016; Wittmann et al. 2017; Parsa et al. 2018), we reasoned that inserting a cluster of Seb1-binding sites downstream from the proximal PAS would increase the usage of the proximal site, thereby promoting premature termination of $n c$-tgp 1 transcription. We therefore used genome editing by CRISPR/Cas9 to insert five consensus Seb1-binding sites downstream from the proximal PAS of $n c$-tgp1 (Fig. 3D, see $p P A S+5 s e b 1$ ). Notably, the insertion of Seb1-binding motifs downstream from the proximal nc-tgp1 PAS resulted in increased tgp1 expression together with reduced levels of $n c-\operatorname{tg} p 1$ lncRNA (Fig. 3E). Thus, by imposing RNAPII pausing downstream from the proximal nc-tgp1 PAS, thereby promoting $3^{\prime}$ end processing and transcription termination, tgp 1 expression was induced even in phosphate-rich conditions.

\section{Recruitment of the Pho7 transcription factor at the tgp1} promoter is increased in the RNAPII slow mutant

It was previously noted that the distal PAS of nc-tgp1 coincides with a binding site in the $\operatorname{tgp} 1$ promoter for $\mathrm{Pho} 7$ (Sanchez et al. 2018a), a key transcription factor that controls phosphate homeostasis in fission yeast. Such overlap between the lncRNA transcription unit and the tgp1 promoter argues for transcriptional interference (Ard et al. 2014; Ard and Allshire 2016). Accordingly, premature transcription termination of the $n c$ - $\operatorname{tgp} 1$ lncRNA gene in the RNAPII slow mutant (Figs. 2, 3) is expected to provide accessibility for Pho7 recruitment at the $\operatorname{tgp} 1$ promoter. To examine Pho7 binding at the tgp 1 promoter in wild- 

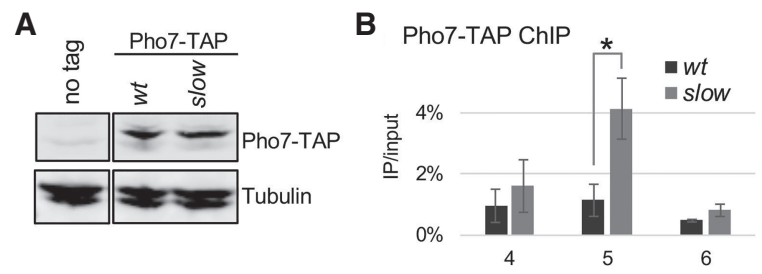

Figure 4. Increased recruitment of $\mathrm{Pho} 7$ at the $\operatorname{tgp} 1$ promoter in the $r p b 1$ slow mutant. $(A)$ Western blot analysis of TAP-tagged Pho7 in the wild-type and slow mutant with tubulin as a loading control. The image for the no-tag control strain was cropped from the same blot as the other two samples. $(B)$ ChIP analysis of Pho7TAP binding at the $\operatorname{tgp} 1$ promoter (amplicons 4-6, as described in Figs. 2, 3) in the wild-type and slow mutant. The error bars represent standard deviation of the mean from three independent experiments. The difference between the slow and wild-type strains is significant at region 5 (two-sided Student's $t$-test $P$-value $=$ 0.0191 .

type and rpb1-N494D cells, we analyzed chromatin occupancy of a TAP-tagged version of Pho7 (Fig. 4A) by ChIP assays. Despite equal expression of Pho7-TAP in both strains (Fig. 4A), there was a fourfold increase in Pho7 occupancy at the $\operatorname{tgp} 1$ promoter in the RNAPII slow mutant relative to the wild-type control (Fig. 4B, see region 5). We therefore conclude that induction of the $\operatorname{tgp} 1 \mathrm{mRNA}$ in the RNAPII slow mutant (Fig. 1) is the direct consequence of Pho7-dependent transcriptional activation.

\section{Premature termination of nc-tgp1 transcription triggers} tgp1 induction upon phosphate starvation

The aforementioned results suggest a model whereby the slower elongation rate of the Rpb1 N494D mutant favors the cotranscriptional utilization of proximal PASs in $n c$ $\operatorname{tgp} 1$, thereby promoting premature termination of $n c$ $\operatorname{tg} p 1$ transcription, which in turn allows for $\operatorname{tg} p 1$ promoter activation by the Pho7 transcription factor (see Fig. 5A). To examine whether premature termination of nc-tgp1 transcription is used to activate $\operatorname{tgp} 1$ upon nutrient starvation, we analyzed tgp1 expression in phosphate-rich $\left(+\mathrm{PO}_{4}\right)$ and phosphate-deprived $\left(-\mathrm{PO}_{4}\right)$ conditions using wild-type cells. As expected, the levels of $\operatorname{tg} p 1$ mRNA increased after phosphate starvation (Fig. 5B, amplicons 68). In contrast, levels of the $n c$ - $\operatorname{tg} p 1$ lncRNA were substantially decreased in the absence of phosphate (Fig. 5B, amplicons 1-5). To assess whether the loss of the lncRNA upon phosphate starvation was due to premature transcription termination of $n c-\operatorname{tg} p 1$, we monitored RNAPII occupancy along the $n c$-tgp1 and $\operatorname{tgp} 1$ loci in $+\mathrm{PO}_{4}$ and $-\mathrm{PO}_{4}$ conditions. As shown in Figure 5C, Rpb1 levels declined downstream from the proximal PAS in phosphatestarved cells relative to cells grown in phosphate-rich conditions (regions 2-5), consistent with premature termination of $n c-\operatorname{tgp} 1$ transcription induced by a phosphate deficiency. In agreement with this, the levels of Rna14 and Seb1 $3^{\prime}$ end processing/transcription termination factors were both redistributed toward the proximal PAS of $n c$ tgp1 in phosphate-deficient cells (Fig. 5D,E). Therefore, in conditions of phosphate limitation, transcription is prematurely terminated at the nc-tgp1 locus to allow for induction of $\operatorname{tgp} 1$ expression (see model in Fig. 5A).

\section{Widespread changes in alternative polyadenylation (APA) in the RNAPII slow mutant}

Most genes in eukaryotes contain multiple cleavage/polyadenylation sites, which can be dynamically regulated during cell proliferation and differentiation as well as in response to various stresses (Mayr 2016; Tian and Manley 2017). Our data support that APA coupled to transcription termination of an upstream lncRNA gene is the mechanism responsible for tgp1 activation in the RNAPII slow mutant as well as in conditions of phosphate starvation in fission yeast. To measure the global effect of a slow
A
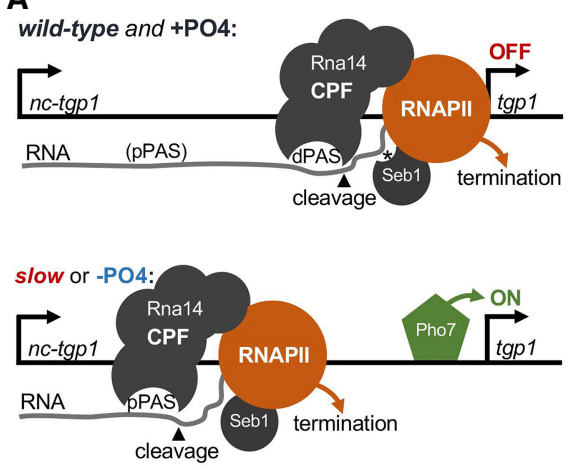

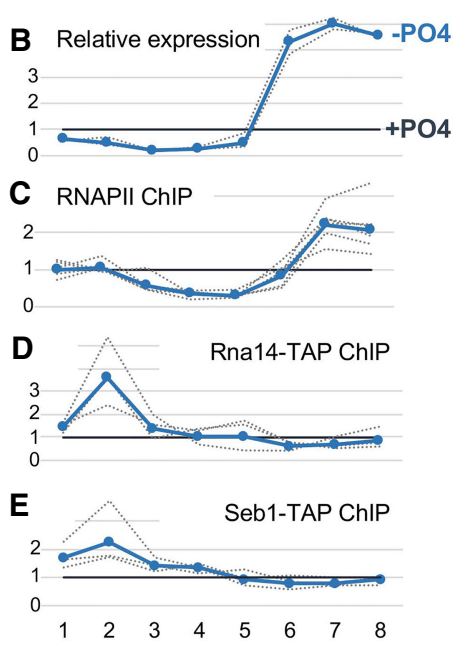

Figure 5. Phosphate-dependent regulation of $\operatorname{tgp} 1$ expression is controlled by premature termination of $n c$ - $\operatorname{tg} p 1$ transcription. (A) Model for $\operatorname{tg} p 1$ regulation by alternative cleavage and polyadenylation of its upstream noncoding RNA nc-tgp1 (see text for details). (*) seb1-binding site; (CPF) cleavage and polyadenylation factor complex. $(B-E)$ Wild-type cells grown in phosphate-containing minimal media $(+\mathrm{PO} 4)$ were shifted into either $+\mathrm{PO} 4$ or phosphate-free $(-\mathrm{PO} 4)$ minimal media during $4 \mathrm{~h}$ for all experiments. The results are expressed relative to the $+\mathrm{PO} 4$ condition after the 4$\mathrm{h}$ incubation. Individual replicates are shown as dashed lines and their average is displayed as a thick blue line. The amplicons 1-8 target the $n c$ $\operatorname{tgp} 1 / \operatorname{tgp} 1$ locus, as described in Figures 2 and 3. (B) RT-qPCR analysis of $n c$-tgp1/tgp1 expression upon phosphate depletion from two independent experiments. $(C-E)$ Relative chromatin occupancy of Rpb1 $(C)$, Rna14 $(D)$, and Seb1 $(E)$ at the $n c$ -

tgp1/tgp1 locus upon phosphate depletion from six $(C)$ or three $(D, E)$ independent experiments. Individual replicates were normalized to total RNAPII levels, as measured from the same chromatin preparation. 
transcription elongation rate on APA, we examined the changes in poly(A) site selection from our transcriptomic data of the slow mutant and control strain for genes with at least two independent PASs $(n=2208)$. Specifically, we calculated a distal/proximal ratio from the RNA-seq read coverage for genomic regions based on proximal and distal PAS usage (Liu et al. 2017b) and compared this ratio between the wild-type and the RNAPII slow mutant to get an APA score. In total, we found 516 genes that showed a significant change in APA score in the slow mutant relative to wild-type cells (Fig. 6A; Supplemental Table S2). Notably, of the 516 APA-sensitive genes identified in the RNAPII slow mutant, 441 (85\%) showed a shift toward increased usage of the proximal PAS, including the nc-tgp1 lncRNA (Fig. 6A, red dot). Most of the 441 APA events that showed a distal-to-proximal shift in the slow mutant took place in the $3^{\prime}$ UTR of protein-coding genes (Fig. 6B), as shown for SBPC16E9.15 (Fig. 6C) and tim11 (Fig. 6D). We also found cases in which the RNAPII slow mutant showed reduced read coverage downstream from poly(A) sites clustered in the $5^{\prime}$ UTR of genes (Fig. 6E), suggesting that premature transcription termination close to transcription start sites is used to control gene expression in fission yeast.

Altogether, we found that $20 \%$ (441 out of 2208) of genes with at least two independent PASs showed a shift toward the preferential use of the proximal PAS in the rpb1 slow mutant (Fig. 6A,B). To examine the possibility that differences in the sequences surrounding the affected PAS could explain why a subset of genes are particularly sensitive to a reduction in transcription elongation rate, DREME (Bailey 2011) was used to compare motif sequences $40 \mathrm{nt}$ upstream of and downstream from the proximal cleavage sites of affected and unaffected genes. No significant differences were observed upstream of the cleavage sites, but a U-rich motif (Fig. 6F) was found to be differentially enriched $\left(e\right.$-value $\left.=3.5 \times 10^{-2}\right)$ downstream from the cleavage sites of affected genes. Analysis of nucleotide frequencies also revealed that the regions immediately upstream of and downstream from the A-rich cleavage sites of proximal PASs of genes affected by the slow mutant were significantly enriched in uridine $(P=3.084 \times$ $10^{-8}$ ) compared with proximal PAS of unaffected genes (Supplemental Fig. S8). These observations support the notion that specific genes tend to share certain sequence motifs surrounding the proximal PAS, which may allow them to be particularly sensitive to a reduction in transcription elongation rate.

We next examined the connection between APA-sensitive events and changes in gene expression. We observed a significant overlap between genes that showed distal-toproximal APA and those that were up-regulated in rpb1N494D cells (Fig. 6G). Within this overlapping module, computational analyses (Bitton et al. 2015) to examine for enrichment of functional gene classes revealed that genes involved in the purine nucleotide biosynthesis
A

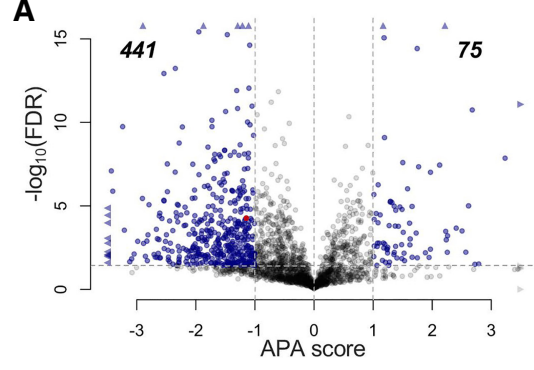

C

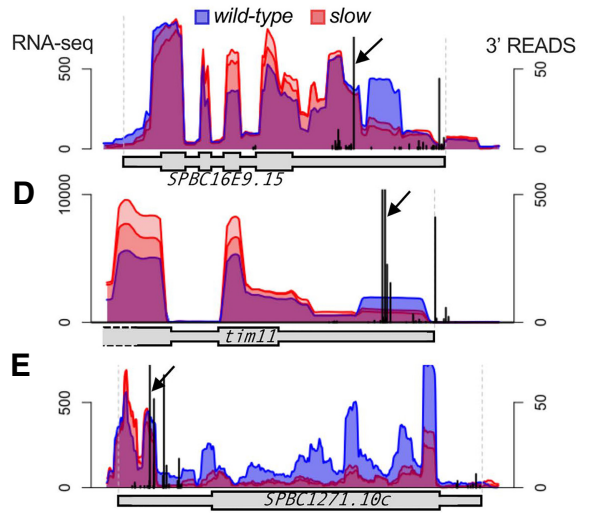

B

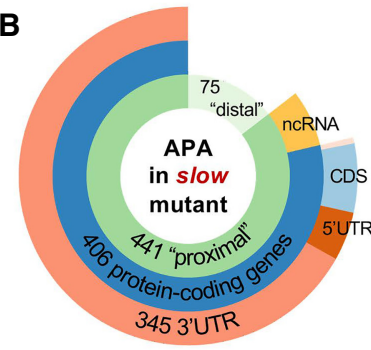

$\mathbf{F}$

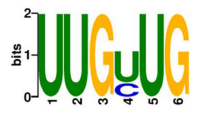

G

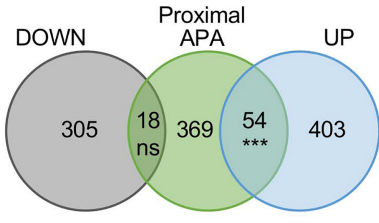

$\mathbf{H}$

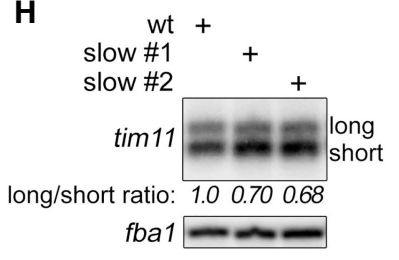

Figure 6. Widespread changes in poly(A) site selection in the rpb1 slow mutant. (A) Volcano plot of statistical significance $\left(-\log _{10}\right.$ of false discovery rate, FDR) against the APA score defined as the $\log _{2}$ ratio of distal to proximal segment ratio between the slow mutants and the wild-type $\mid n=$ 2208 genes with at least two independent PASs) (see the Materials and Methods). Dashed lines represent the thresholds to select significant APA events (in blue); absolute APA score $>1$ and FDR $<0.01$. The single red dot represents $n c-\operatorname{tgp} 1$. To ease viewing, genes with values beyond axes limits are represented by arrowheads. $(B)$ Distribution of the significant APA events identified among gene features. The terms "proximal" and "distal" APA describe genes for which the slow mutation favors proximal (negative APA score) and distal (positive APA score) PAS usage, respectively. $(C-E)$ Normalized read coverage (left axis) at three different loci for the wild-type (blue area) and slow mutants (red areas) from RNA-seq data, and for previously published 3' READS data (Liu et al. 2017b) in wildtype cells (black peaks; right axis). Black arrows indicate the proximal PAS at which the read coverage decreases in the slow mutant. Transcription is $5^{\prime}$ to $3^{\prime}$, left to right. $(F)$ Sequence logo of UUG[UC]UG motif enriched downstream from affected versus unaffected proximal PAS. (G) Overlap between genes for which the RNAPII slow mutant significantly favors proximal (APA score $<-1)$ poly(A) sites and the genes significantly down-regulated and up-regulated in the slow mutant. $\left(^{* * *}\right) P$-value $=4.98 \times 10^{-06}$; (ns) ot significant. $(H)$ Northern blot analysis using a probe specific to tim 11 highlighting the long (634-nt) and short (539-nt) isoforms. The average ratio $(n=3)$ between both isoforms, normalized to the wild-type parental strain, is indicated in italic below each lane. 
process were significantly over-represented ( $P$-value $5 \times$ $10^{-06}$; odds ratio 2.15). An example of this is shown for tim11, a gene encoding a subunit of the F1-FO ATP synthase, for which we noted decreased read coverage downstream from the proximal poly(A) site together with a twofold increase in read coverage inside the gene body in the slow mutant (Fig. 6D). The effect of the RNAPII slow mutant on tim11 poly(A) site selection was confirmed by Northern blotting (Fig. 6H). Our data thus indicate that the slow transcription elongation rate in $r p b 1$ N494D cells causes widespread distal-to-proximal shifts in APA, affecting the expression of a specific set of genes, including the core $\mathrm{PHO}$ regulon (indirectly via upstream lncRNA) and genes involved in the purine biosynthesis pathway (via 3' UTR shortening).

\section{Limiting nucleoside triphosphate concentration activates phosphate-responsive genes}

Our results reveal that fission yeast respond to conditions of both phosphate starvation and slow transcription elongation by inducing the expression of a core $\mathrm{PHO}$ regulon via a mechanism that involves APA and premature transcription termination of interfering lncRNAs. To elucidate the molecular events that elicit this gene activation response, we considered that a deficiency in inorganic phosphate results in depletion of the intracellular NTP pool (Boer et al. 2010; Ljungdahl and Daignan-Fornier 2012). Notably, when NTP substrate concentrations are reduced by treating cells with drugs such as 6AU and MPA that impair nucleotide biosynthesis (Exinger and Lacroute 1992; Shaw and Reines 2000), the elongation rate of RNAPII decreases (Mason and Struhl 2005). Thus, we reasoned that $\operatorname{tgp} 1$, pho1, and pho84 genes may have evolved to be positively regulated by a reduced rate of transcription elongation, which is anticipated in conditions of phosphate starvation as a consequence of limiting NTP concentration. To test whether limiting NTP concentration results in the activation of the core
PHO regulon, we treated wild-type cells with 6AU and MPA. As shown in Figure 7, A and B, 6AU and MPA both resulted in increased levels of the core PHO regulon genes, although the effect of MPA on pho84 expression was minimal. NTP depletion using 6AU or MPA also resulted in increased levels of the short $3^{\prime}$ UTR version of tim 11 relative to the long $3^{\prime}$ UTR isoform (Fig. 7C), consistent with increased usage of proximal poly(A) sites as a consequence of reduced transcription elongation rates. Our results thus support the notion that decreased intracellular NTP concentrations as a consequence of nutrient limitations can result in an overall reduction in the transcription elongation rate, which in turn can activate genes that are particularly sensitive to slower RNAPII kinetics, such as genes that promote immediate phosphate uptake (core PHO regulon) and purine biosynthesis.

\section{Discussion}

It is now widely appreciated that the rate of RNAPII elongation is highly dynamic, varying extensively both within and between genes (Danko et al. 2013; Jonkers et al. 2014; Veloso et al. 2014; Cortazar et al. 2019). To date, however, how the transcription elongation rate is controlled still remains poorly understood. Furthermore, it remains unknown whether changes in the elongation rate of RNAPII are used to control and coordinate gene expression. In this study, we used a previously characterized $r p b 1$ mutant (Malagon et al. 2006; Jimeno-González et al. 2010; Strathern et al. 2013) to investigate how a slow transcription rate globally affects gene expression. Intuitively, a reduced rate of transcription elongation is expected to result in a general decrease in mRNA synthesis. However, although our RNA-seq analyses revealed a set of genes with significantly reduced RNA accumulation, the large majority of genes showed normal steady-state RNA levels in the RNAPII slow mutant (Fig. 1). This can be explained, at least in part, by a general decrease in RNA decay rates,
A

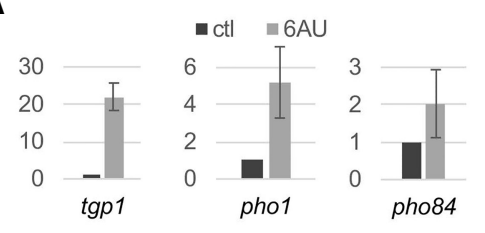

C

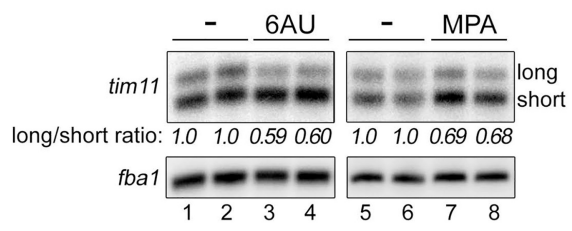

B

D

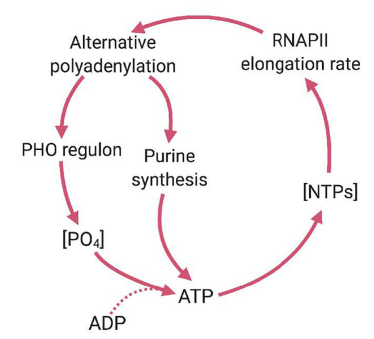

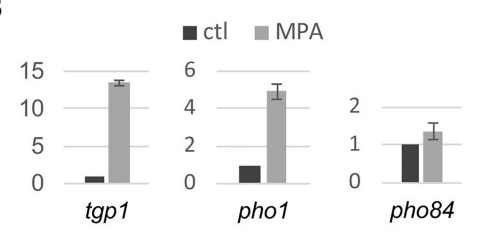

Figure 7. Limiting NTP concentration activates phosphate-responsive genes. $(A, B)$ RT-qPCR analysis of tgp1, pho1, and pho84 expression relative to nda2 in response to 6AU and MPA. Cells were grown in EMM and treated for $1 \mathrm{~h}$ with either $30 \mu \mathrm{g} / \mathrm{mL}$ of 6 -azauracil (6AU) (A) or $30 \mu \mathrm{g} / \mathrm{mL}$ of mycophenolic acid (MPA) (B) or left untreated (ctl). Error bars represent the standard deviation of the mean over two independent experiments. $(C)$ Northern blot analysis of tim 11 short and long isoforms. Total RNA prepared from wild-type cells that were treated with 6AU (lanes 3,4) or MPA (lanes 7,8 ), as described in $A$ and $B$, was analyzed by Northern blotting. Control cells were treated with DMSO (lanes 1,2) or methanol (lanes 5,6). Independent replicates are shown (cf. lanes 1 and 3, 2 and 4, 5 and 7, and 6 and 8 ). The average ratio between both isoforms, normalized to the ratio in untreated control cells, is indicated in italic below each lane. $(D)$ Model of homeostatic regulation of phosphate-responsive genes by control of RNAPII elongation kinetics and APA (see the text for details). 
which apparently compensates for the decreased rate of mRNA synthesis in this $r p b 1$ slow mutant (Sun et al. 2012). On the other hand, the up-regulation of more than 450 transcripts (Fig. 1), including a core set of genes involved in phosphate acquisition and purine synthesis, in a mutant with a reduced rate of transcription elongation was mostly unexpected. By studying how the expression of the phosphate-responsive permease gene $\operatorname{tgp} 1$ is up-regulated in the rpb1 slow mutant, we unveiled a mechanism that involves alternative polyadenylation (APA) and premature transcription termination of an upstream interfering lncRNA (Fig. 5A) and showed that this mechanism is occurring naturally to activate $\operatorname{tgp} 1$ expression in conditions of phosphate starvation. Importantly, our findings argue that variations in the transcription elongation rate is a critical mode by which cells control their transcriptome in response to nutrient limitations (Fig. 7D).

\section{Transcription elongation kinetics regulate alternative polyadenylation (APA)}

Most eukaryotic genes harbor multiple poly(A) sites and a growing number of mechanisms have been shown to control APA, including core 3' end processing factors and specific RNA-binding proteins (Mayr 2017; Tian and Manley 2017). Previous findings in Drosophila suggest that kinetic competition between RNAPII elongation and $3^{\prime}$ end processing influences poly(A) site selection (Pinto et al. 2011; Liu et al. 2017a). Mechanistically, RNAPII speed would regulate APA by restraining the competition between alternative PASs: A slow elongation rate would favor proximal PAS usage by increasing the window of time that this PAS is available for cleavage by the $3^{\prime}$ end processing machinery before a competing distal PAS is transcribed. Our RNA-seq analysis of the rpb1-N494D mutant is totally consistent with this view, revealing that a reduction in the transcription elongation rate enhances proximal PAS usage (Fig. 6). It therefore appears that RNAPII elongation kinetic is a critical parameter that influences the efficiency of most cotranscriptional RNA processing events, including splicing (Roberts et al. 1998; de la Mata et al. 2003; Fong et al. 2014; Aslanzadeh et al. 2018), poly(A) site selection (this study; Pinto et al. 2011; Liu et al. 2017a), and transcription termination (Hazelbaker et al. 2013; Fong et al. 2015; Cortazar et al. 2019).

\section{Gene activation by APA-dependent premature termination of IncRNA transcription}

In yeast, a conserved set of phosphate-responsive genes (core PHO regulon) provides the initial response to phosphate starvation by rapidly inducing pho1, pho84, and tgp1 (Carter-O'Connell et al. 2012), whose protein products are responsible for immediately harvesting inorganic phosphate from the environment. Interestingly, these three genes have been shown to be repressed by upstream lncRNA-mediated transcription in fission yeast (Ard et al. 2014; Shah et al. 2014; Garg et al. 2018; Sanchez et al. 2018a). In recent years, there has been an increasing number of examples of negative gene regulation by adjacent transcription of a lncRNA, a process often referred to as transcriptional interference, which has been described in yeast, fruit flies, mice, and humans (Ard et al. 2017). Transcriptional interference generally depends on refractory changes caused by the transcription of an upstream noncoding RNA in the chromatin architecture of a downstream protein-coding gene promoter. Although numerous mechanisms whereby the act of lncRNA transcription represses a downstream gene have been described (Ard et al. 2017; Kaikkonen and Adelman 2018), how these systems are reversibly inactivated to allow induction of the protein-coding gene has remained poorly understood. In this study, we found that the core $\mathrm{PHO}$ regulon in $S$. pombe is activated by a mutant RNAPII with reduced elongation rate. Several lines of evidence support that activation of $\operatorname{tgp} 1$, a core $\mathrm{PHO}$ regulon gene in $S$. pombe, is mediated by premature termination of upstream lncRNA transcription. First, reduction of transcription elongation rate in the rpb1-N494D mutant caused a decline in RNAPII occupancy beyond the proximal nc-tgp1 PAS, which coincided with reduced production of nc-tgp1 lncRNA (Fig. 2), increased Pho7 binding at the $\operatorname{tgp} 1$ promoter (Fig. 4), and induction of $\operatorname{tgp} 1$ mRNA (Figs. 1,2). Second, slower RNAPII elongation resulted in the redistribution of $3^{\prime}$ end processing/termination factors from the distal to the proximal PAS of the nc-tgp1 lncRNA (Fig. 3). Third, enforcing RNAPII pausing and transcription termination at the proximal nc-tgp1 PAS was sufficient to induce tgp1 expression in phosphate-rich conditions (Fig. 3D,E). Finally, a mutation altering the proximal poly(A) signal of $n c-\operatorname{tgp} 1$ was shown to attenuate $\operatorname{tgp} 1$ induction (Sanchez et al. 2018a).

Collectively, these findings support a model of gene regulation by lncRNA-mediated transcriptional interference that is controlled by APA and premature termination of lncRNA transcription (Fig. 5A). In wild-type cells, an optimal rate of transcription elongation together with the absence of Seb1-binding motifs downstream from the proximal PAS results in suboptimal utilization of the proximal PAS relative to the distal PAS during transcription of $n c-\operatorname{tg} 1$ (Fig. 5A, top panel). As the distal nc-tgp1 PAS directly overlaps with the binding site for the transcription factor Pho7 (Schwer et al. 2017), transcription and $3^{\prime}$ end processing at the distal nc-tgp1 PAS is likely to impinge on the binding of $\mathrm{Pho} 7$ to the $\operatorname{tgp} 1$ promoter. Transcription-coupled chromatin modifications may also contribute to repress $\operatorname{tgp} 1$ expression via lncRNA transcription through its promoter, a mechanism shown previously for tgp1 (Ard and Allshire 2016) and other fission yeast genes (Shah et al. 2014; Touat-Todeschini et al. 2017). In the context of a slower transcription elongation rate, such as in rpb1-N494D cells, the frequency of proximal PAS usage is increased relative to the distal PAS, leading to premature termination of nc-tgp1 transcription, which prevents lncRNA transcription through the downstream tgp1 promoter, thereby allowing for the recruitment of the Pho7 transcription factor and tgp 1 induction (Fig. 5A, bottom panel). 
Importantly, we also show that phosphate-dependent regulation of $\operatorname{tgp} 1$ expression is controlled by APA and premature termination of the upstream $n c$ - $\operatorname{tg} p 1$ lncRNA (Fig. 5). This is in contrast to previous examples of lncRNA-mediated gene regulation where the expression of the lncRNA is regulated at the level of transcriptional initiation (Martens et al. 2004, 2005; Hirota et al. 2008). Indeed, our ChIP data indicate similar levels of RNAPII at the nc$\operatorname{tgp} 1$ promoter between the wild-type and the slow mutant (Fig. 2D, see region 1) as well as between phosphate-rich and phosphate-deficient conditions (Fig. 5C, region 1). Thus, although we cannot completely rule out some level of transcriptional control of the $n c$ - $\operatorname{tgp} 1$ lncRNA promoter in response to phosphate concentration, our results argue that APA-dependent premature termination of noncoding RNA transcription is a crucial mechanism of gene activation for the core PHO regulon in S. pombe.

While premature $3^{\prime}$ end processing is a widespread phenomenon in the RNAPII slow mutant (Fig. 6), our RNAseq data indicated that a small number of ncRNAs $(n=$ $35)$ relative to protein-coding genes $(n=406)$ showed premature $3^{\prime}$ end processing. Although nc-tgp1/tgp1 and prt2/pho84 provide strong cases where premature termination of an upstream interfering ncRNA affects the expression of a downstream gene, manual inspection of the remaining ncRNAs affected in the RNAPII slow mutant did not provide clear evidence for activation of a neighboring protein-coding gene. However, it remains possible that regulation of transcription elongation rate at ncRNA genes may modulate the expression of adjacent protein-coding genes by other mechanisms than premature termination. For instance, the RNA-seq analysis of the RNAPII slow mutant revealed the induction of ste11, which encodes a master transcription factor for sexual development that activates a number of genes required for mating and meiosis in fission yeast. Interestingly, similar to the core PHO regulon genes, ste 11 is induced by nutrient starvation and is negatively regulated by a lncRNA. In the case of ste11, gene repression is mediated in cis by a divergently transcribed lncRNA, rse 1, that recruits a protein complex that promotes deacetylation of the ste 11 promoter (Fauquenoy et al. 2018). As a secondary structure in the divergently transcribed rse 1 lncRNA appears to tether the repressive complex that deacetylates the ste11 promoter (Fauquenoy et al. 2018), it is tempting to speculate that RNAPII elongation rate could influence the efficiency of cotranscriptional lncRNA folding, which can have crucial consequences on the recruitment of RNA-binding proteins. Consistent with this view, the speed of transcription elongation has been shown to affect riboswitch folding in bacteria (Wickiser et al. 2005; Chauvier et al. 2017) and formation of a stem-loop structure important for histone pre-mRNA processing in humans (Saldi et al. 2018).

Transcription elongation rate controls a gene expression program in response to phosphate starvation

An unexpected finding from our study was that the preferential use of proximal PASs in the RNAPII slow mutant resulted in increased expression of genes involved in two important and related cellular pathways: phosphate acquisition and purine biosynthesis. What is the physiological relevance of decreased transcription speed increasing the expression of the core $\mathrm{PHO}$ regulon and genes involved in purine biosynthesis? Interestingly, purine and phosphate pathways are known to be coregulated in budding and fission yeasts (Gauthier et al. 2008; Estill et al. 2015), but the underlying mechanism linking the two pathways has remained unclear. A key role of phosphate assimilation in cells is the phosphorylation of ADP to ATP, a purine required for nucleic acid synthesis and a central energy supply (Ljungdahl and Daignan-Fornier 2012). Accordingly, phosphate starvation in yeast causes a drastic deficiency in ATP, but also in UTP and CTP (Boer et al. 2010). Since these NTPs are direct substrates of RNAPII during RNA synthesis, limiting intracellular NTP concentrations causes a decrease in the rate and processivity of RNAPII (Mason and Struhl 2005). Our data therefore support a model (Fig. 7D) whereby nutrient availability modulates the elongation rate of RNAPII to control the activation of specific gene expression programs. In the case of phosphate starvation, we propose that limitation for extracellular phosphate results in intracellular NTP depletion, causing a global reduction in transcription elongation rate. Importantly, we have shown that a reduction in RNAPII elongation rate enhances proximal PAS usage by APA, resulting in activation of genes specifically needed in response to phosphate starvation: the core $\mathrm{PHO}$ regulon and genes involved in purine biosynthesis (Fig. 7D). Interestingly, links between the nutritional status of the cell, NTP concentration, and transcription have been previously established in budding yeast. Accordingly, NTP sensing by the transcription machinery provides a mechanism to control the expression of genes involved in specific nucleotide biosynthesis pathways; however, in this case, limiting NTP concentration control RNAPII initiation at specific promoters (Davis and Ares 2006; Thiebaut et al. 2008; Kuehner and Brow 2019). The identification of a mechanism of gene activation that responds to nutrient limitation by altering RNAPII elongation kinetics, as reported here, thus constitutes a new regulatory paradigm that could allow cells to meet the rapidly changing needs in nutrient availability. Quite remarkably, our results therefore suggest that subsets of genes have evolved specific mechanisms that allow them to be particularly responsive to a slow transcription elongation rate. For the core $\mathrm{PHO}$ regulon genes, we provide a molecular explanation for how APA-mediated premature termination of upstream lncRNA transcription allow for their activation in cells with a reduced rate of RNAPII elongation. In the case of genes involved in purine biosynthesis, future work will determine how 3' UTR shortening of encoded mRNAs can lead to increased expression upon reduced RNAPII elongation kinetics.

In summary, our findings support the notion that the nutritional state of eukaryotic cells, reflected by NTP concentrations, can alter the rate and processivity of RNAPII elongation, which in turn influence the efficiency of RNA processing events such as poly(A) site selection to regulate 
specific expression programs. Given the primordial role of alternative splicing and alternative polyadenylation in mammalian cells, it will be interesting to see whether variations in the RNAPII elongation rate in responses to changes in the nutritional status or the during cellular differentiation can also activate specific expression programs in higher eukaryotes.

\section{Materials and methods \\ Yeast strains and media}

A list of all S. pombe strains used in this study is provided in Supplemental Table S3. Unless stated otherwise, cells were grown to mid-log phase $\left(\mathrm{OD}_{600} 0.5-0.7\right)$ at $30^{\circ} \mathrm{C}$ in YES or EMM medium supplemented with adenine, histidine, leucine, and uracil. CRISPR/Cas9-mediated mutagenesis was used to generate the rpb1-N494D and +5seb1 mutants by applying the "fluoride-selection" (Fernandez and Berro 2016) or the "short homology" protocol (Hayashi and Tanaka 2019), respectively. C-terminal tagging of proteins were performed by PCR-mediated gene targeting (Bähler et al. 1998) using lithium acetate method for yeast transformation.

\section{Growth assays}

Mycophenolic acid (MPA; Sigma m3536) and 6-azauracil (6AU; Sigma A1757) were resuspended in methanol and DMSO, respectively, to a stock concentration of $50 \mathrm{mg} / \mathrm{mL}$. As the large quantity of uracil contained in uracil-supplemented media neutralizes the effect of 6AU (Reines 2003), the tested S. pombe strains were made autotroph for uracil by transformation with ura4-containing plasmids (FB168). Exponentially growing cultures of those ura4+ strains were concentrated to an $\mathrm{OD}_{600}: 1$ and serially diluted fivefold in the appropriate liquid media. Each dilution was spotted on freshly prepared plates containing the indicated concentration of drug or an equal volume of solvent.

\section{Western blots}

Total cell extracts were prepared by harvesting cells in mid-log phase in ice-cold lysis buffer $(50 \mathrm{mM}$ Tris at $\mathrm{pH} 7.5,5 \mathrm{mM}$ $\mathrm{MgCl} 2,150 \mathrm{mM} \mathrm{NaCl}$ and $0.1 \% \mathrm{NP}-40$ ) containing a cocktail of protease inhibitors $(1 \times$ PMSF, $1 \times$ PLAAC) with half of a phosphor STOP tablet, prior to lysis with glass beads using a FastPrep instrument (MP Biomedicals). Clarified lysates were normalized for total protein concentration using the Bradford protein assay. Thirty micrograms of total proteins was separated by SDSPAGE, transferred to nitrocellulose membranes, and analyzed by immunoblotting using a rabbit polyclonal antibody against protein A (1:10,000 [v/v] dilution; Sigma-Aldrich, P3775). Membranes were then probed with goat anti-rabbit secondary antibodies conjugated to IRDye $800 \mathrm{CW}$ (1:15,000 [v/v] dilution; LI-COR, 926-32213). Detection of the proteins was performed using an Odyssey infrared imaging system (LI-COR).

\section{Chromatin immunoprecipitation (ChIP) assays}

For each IP, $50 \mathrm{~mL}$ of cells grown in EMM were incubated for 20 min at room temperature with $1 \%$ formaldehyde. After quenching the reaction with glycine (final concentration of $360 \mathrm{mM}$ ) for $5 \mathrm{~min}$ at room temperature, cells were washed twice with cold Tris-buffered saline $(20 \mathrm{mM}$ Tris- $\mathrm{HCl}$ at $\mathrm{pH} 7.5,150 \mathrm{mM}$ $\mathrm{NaCl}$ ). Cell pellets from $50-\mathrm{mL}$ cultures were resuspended in
$500 \mu \mathrm{L}$ of lysis buffer (50 mM HEPES-KOH at $\mathrm{pH} 7.5,140 \mathrm{mM}$ $\mathrm{NaCl}, 1 \mathrm{mM}$ EDTA at pH 8.0, $1 \%$ Triton X-100, 0.1\% Na-deoxycholate) containing protease inhibitors and disrupted using a FastPrep instrument. Samples were then sonicated 10 times for $10 \mathrm{sec}$ at $20 \%$ intensity using a Branson digital sonifier. Wholecell extract $\left(\mathrm{WCE}_{;} 500 \mu \mathrm{L}\right)$ was incubated overnight at $4^{\circ} \mathrm{C}$ with $50 \mu \mathrm{L}$ of magnetic beads only (PAN mouse IgG; Invitrogen) in the case of the immunoprecipitation of HTP/TAP-tagged proteins, or of magnetic beads coupled with $2 \mu$ g of one of the following antibodies: 8WG16 (unphosphorylated CTD), clone 3E10 (phospho CTD Ser2; Millipore), clone 3E8 (phospho CTD Ser5; Millipore), clone 3D4A12 (phospho CTD Ser7; Active Motifs), and clone 6D7 (phospho CTD Thr4; Active Motifs). Beads were washed twice with $1 \mathrm{~mL}$ of lysis buffer, twice with $1 \mathrm{~mL}$ of lysis buffer plus $500 \mathrm{mM} \mathrm{NaCl}$, twice with $1 \mathrm{~mL}$ of wash buffer $(10 \mathrm{mM}$ Tris- $\mathrm{HCl}$ at $\mathrm{pH} 8.0,250 \mathrm{mM} \mathrm{LiCl}, 0.5 \% \mathrm{NP}-40,0.5 \%$ sodium deoxycholate, and $1 \mathrm{mM}$ EDTA), and once with $1 \mathrm{~mL}$ of TrisEDTA (TE; 10 mM Tris- $\mathrm{HCl}$ at pH 8.0, 1 mM EDTA). Bound material was eluted by resuspending beads in $50 \mu \mathrm{L}$ of elution buffer (50 mM Tris-HCl at $\mathrm{pH} 8.0,10 \mathrm{mM}$ EDTA, 1\% SDS) after 15-min incubation at $65^{\circ} \mathrm{C}$. After overnight incubation at $65^{\circ} \mathrm{C}$ for reverse-crosslinking, the samples were treated with proteinase $\mathrm{K}$ and cleaned up by phenol-chloroform extraction before undergoing a RNase treatment and a final purification on PCR purification column (Qiagen). DNA from the inputs and IPs were analyzed on a LightCycler 96 Instrument using perfecta SYBR supermix in the presence of a $150 \mathrm{nM}$ concentration (each) of gene-specific primers in $15-\mu \mathrm{L}$ reaction mixtures. Protein occupancy was then calculated using the percent input method (Lemay et al. 2014; Larochelle et al. 2018). For the IPs of phosphorylated CTD or the RNAPII-associated 3' end processing/termination factors (Rna14 and Seb1), the values were further normalized to RNAPII occupancy measured from the same chromatin preparation, as previously described (Lemay et al. 2016; Larochelle et al. 2018).

\section{$R N A$ preparation and analyses}

Total RNA was extracted using the hot-acid phenol method (Lemay et al. 2014; Larochelle et al. 2018). For Northern blots, RNA samples were resolved on $2 \%$ agarose gels, transferred onto nylon membranes, and probed using ${ }^{32} \mathrm{P}$-labeled riboprobes. For RT-qPCR analyses, RNA samples ( $1 \mu \mathrm{g}$ of total RNA) were treated with DNase RQ1 (Promega, M6101) and reverse transcribed using the Omniscript RT (Quiagen). Gene expression relative to the appropriate control strain was measured on a LightCycler 96 system (Roche) with the $\Delta \Delta C T$ method using the gene nda2 as internal reference (Lemay et al. 2014; Larochelle et al. 2018). RNase protection assays were performed as previously described (Mercier et al. 2008). T7 promoter-containing PCR products were used to produce antisense RNA probes that served to determine nc-tgp1 and act1 RNA levels. ${ }^{32} \mathrm{P}$-labeled antisense RNA probes were produced using the aforementioned PCR products with the use of $\left[\alpha^{-32} \mathrm{P}\right] \mathrm{UTP}$ and T7 RNA polymerase. The act1 transcript was used as an internal control for normalization during quantification of the RNase protection products.

\section{Library preparation and Illumina sequencing}

Library preparation was performed at the RNomics Platform of Université de Sherbrooke. Briefly, $10 \mu \mathrm{g}$ of total RNA extracted from cells grown in EMM was treated with DNase on a RNeasy minikit column (Qiagen) according to the manufacturer's protocol. RNA integrity was assessed on a 2100 Bioanalyzer machine (Agilent Technologies). Ribo-depleted RNA was isolated from 
$1.25 \mu \mathrm{g}$ of total RNA using Illumina RiboZero (yeast), as per manufacturer's protocol. Stranded RNA-seq libraries were then built using the SSV21106 kit (Epicentre) from the ribo-depleted RNA with 13 cycles of PCR amplification. After assessment of libraries quality and molarity on a DNA high-sensitivity chip (Agilent), the libraries were pooled and sequenced in paired-end $(2 \times 100$ bp) on a HiSeq 4000 (Illumina) at the McGill University and Génome Québec Innovation Centre (MUGQIC) Sequencing Service with at least $250 \times$ genome depth (>35 millions reads) for each sample.

\section{RNA-seq data analysis}

The reads were trimmed and aligned according to the MUGQIC RNA-seq pipeline (bitbucket.org/mugqic/genpipes/src/master/ pipelines/rnaseq). Briefly, low-quality reads and adaptor sequences were removed with Trimmomatic v0.36 with parameters ILLUMINACLIP:adapters-truseq.fa:2:30:15:8:true TRAILING:30 MINLEN:32 (Bolger et al. 2014). The remaining reads were then aligned to the $S$. pombe genome assembly ASM294v2 with STAR v2.5.1 (Dobin et al. 2013) and summarized at the gene level using featureCounts with options -p -s 2 -P -d 0 -D 1000 -C -T 4 (Liao et al. 2014). Differential gene expression analysis was performed using the DESeq2 R package (v1.24) (Love et al. 2014). The AnGeLi tool (Bitton et al. 2015) was used to assess enrichment in biological processes of the differentially expressed genes identified. Raw and processed data are available on GEO under the accession number GSE140698.

\section{Alternative polyadenylation (APA) analysis}

To estimate the change in poly(A) site selection from transcriptomic data, we first segmented genes in smaller windows delimited by peaks of 3'READS read counts (Liu et al. 2017b), representing PAS usage for a WT strain grown in EMM. The processed $3^{\prime}$ READS data used in this study are accessible under accession number GSM2496939. To be considered in the segmentation process, $3^{\prime}$ READS peaks had to be constituted of more than five reads and account for at least $5 \%$ of a given gene $3^{\prime}$ READS total read coverage. Distinct peaks closer than $50 \mathrm{nt}$ were merged together at the stronger peak position. For genes delimited by at least two segments, we divided the normalized (DESeq2 median ratios method) RNA-seq read coverage of the most distal segment (relative to transcription start site) by the normalized read coverage of the most proximal segment. Then, we compared this ratio between and the RNAPII slow mutants and the wild-type strain to obtain an APA score defined as $\log _{2}[($ slow $/ w t)$ distal $/$ slow $/$ $w t)_{\text {proximal }}$. Statistical significance of the difference in ratios between the slow mutants and the wild-type strain was assessed with a generalized linear model of the binomial family using the $\operatorname{glm}()$ function in R. This method allows one to take into account not only the change in PAS usage ratio, but also the normalized read counts of the numerator and denominator of the ratio, therefore accounting for the magnitude of count values in the calculation of statistical significance.

\section{Motif and sequence analysis}

Affected proximal PAS $(n=441)$ were defined as having an APA score $<1$ and an associated adjusted $P$-value $($ FDR $)<0.01$. Proximal PAS whose APA score is close to 0, within thresholds (between -0.2215 and 0.02215 ) allowing the same number of PAS to be selected (441), served as the unaffected control group. Differential motif analysis was performed using DREME (Bailey 2011) and average base composition was summarized using FastQC.

\section{Acknowledgments}

We thank Beate Schwer, Damien Hermand, and Francois Robert for critical reading of the manuscript, and the sequencing platforms of the McGill University and Génome Québec Innovation Centre; Calcul Québec and Compute Canada provided the computing infrastructure used to analyze the data. This work was supported by funding from the Natural Sciences and Engineering Research Council of Canada (NSERC) to F.B. (RGPIN-201705482). J. Berro and R.F. are supported by National Institutes of Health/National Institute of General Medical Sciences grant R01GM115636. P.-É.J. and C.Y.S. are supported by the Fonds de recherche du Québec-Santé (FRQS). F.B. holds a Canada Research Chair in Quality Control of Gene Expression.

Author contributions: Y.V., C.Y.S., and F.B. conceived the study and the experimental frame. Y.V., C.Y.S., and P.-E.J. planned and designed the pipelines for processing the RNA-seq data. R.F. generated the $r p b 1$ slow mutant with guidance from J. Berro and F.B. Y.V. and C.Y.S. performed most of the experiments with help from M.D. and M.L. J. Beaudoin performed the RPA analysis under guidance of S.L. and F.B. Y.V. and C.Y.S. performed all of the bioinformatics analyses with the help of P.-E.J. Y.V., C.Y.S., and F.B. prepared the figures. F.B. wrote the manuscript with help of C.Y.S. The manuscript was reviewed by all authors.

\section{References}

Ahn SH, Kim M, Buratowski S. 2004. Phosphorylation of serine 2 within the RNA polymerase II C-terminal domain couples transcription and $3^{\prime}$ end processing. Mol Cell 13: 67-76. doi:10.1016/S1097-2765(03)00492-1

Alexander RD, Innocente SA, Barrass JD, Beggs JD. 2010. Splicing-dependent RNA polymerase pausing in yeast. Mol Cell 40: 582-593. doi:10.1016/j.molcel.2010.11.005

Ard R, Allshire RC. 2016. Transcription-coupled changes to chromatin underpin gene silencing by transcriptional interference. Nucleic Acids Res 44: 10619-10630. doi:10.1093/nar/gkw801

Ard R, Tong P, Allshire RC. 2014. Long non-coding RNA-mediated transcriptional interference of a permease gene confers drug tolerance in fission yeast. Nat Commun 5: 5576. doi:10 $.1038 /$ ncomms 6576

Ard R, Allshire RC, Marquardt S. 2017. Emerging properties and functional consequences of noncoding transcription. Genetics 207: 357-367.

Aslanzadeh V, Huang Y, Sanguinetti G, Beggs JD. 2018. Transcription rate strongly affects splicing fidelity and cotranscriptionality in budding yeast. Genome Res 28: 203-213. doi:10 $.1101 /$ gr.225615.117

Bähler J, Wu JQ, Longtine MS, Shah NG, McKenzie A 3rd, Steever AB, Wach A, Philippsen P, Pringle JR. 1998. Heterologous modules for efficient and versatile PCR-based gene targeting in Schizosaccharomyces pombe. Yeast 14: 943-951.doi:10.1002/ (SICI)1097-0061(199807)14:10<943::AID-YEA292>3.0.CO;2-Y

Bailey TL. 2011. DREME: motif discovery in transcription factor ChIP-seq data. Bioinformatics 27: 1653-1659. doi:10.1093/bio informatics/btr261

Bentley DL. 2014. Coupling mRNA processing with transcription in time and space. Nat Rev Genet 15: 163-175. doi:10.1038/ $\operatorname{nrg} 3662$

Bitton DA, Schubert F, Dey S, Okoniewski M, Smith GC, Khadayate S, Pancaldi V, Wood V, Bähler J. 2015. AnGeLi: a tool for the analysis of gene lists from fission yeast. Front Genet 6: 330 . doi: $10.3389 /$ fgene. 2015.00330 
Boer VM, Crutchfield CA, Bradley PH, Botstein D, Rabinowitz JD. 2010. Growth-limiting intracellular metabolites in yeast growing under diverse nutrient limitations. Mol Biol Cell 21: 198-211. doi:10.1091/mbc.e09-07-0597

Bolger AM, Lohse M, Usadel B. 2014. Trimmomatic: a flexible trimmer for Illumina sequence data. Bioinformatics 30: 2114-2120. doi:10.1093/bioinformatics/btu170

Carter-O'Connell I, Peel MT, Wykoff DD, O'Shea EK. 2012. Genome-wide characterization of the phosphate starvation response in Schizosaccharomyces pombe. BMC Genomics 13: 697. doi:10.1186/1471-2164-13-697

Casañal A, Kumar A, Hill CH, Easter AD, Emsley P, Degliesposti G, Gordiyenko Y, Santhanam B, Wolf J, Wiederhold K, et al. 2017. Architecture of eukaryotic mRNA $3^{\prime}$-end processing machinery. Science 358: 1056-1059.

Chauvier A, Picard-Jean F, Berger-Dancause JC, Bastet L, Naghdi MR, Dubé A, Turcotte P, Perreault J, Lafontaine DA. 2017. Transcriptional pausing at the translation start site operates as a critical checkpoint for riboswitch regulation. Nat Commun 8: 13892. doi:10.1038/ncomms13892

Churchman LS, Weissman JS. 2011. Nascent transcript sequencing visualizes transcription at nucleotide resolution. Nature 469: 368-373. doi:10.1038/nature09652

Cortazar MA, Sheridan RM, Erickson B, Fong N, Glover-Cutter K, Brannan K, Bentley DL. 2019. Control of RNA Pol II speed by PNUTS-PP1 and Spt5 dephosphorylation facilitates termination by a sitting duck torpedo' mechanism. Mol Cell 76: 896-908.e4. doi:10.1016/j.molcel.2019.09.031

Danko CG, Hah N, Luo X, Martins AL, Core L, Lis JT, Siepel A, Kraus WL. 2013. Signaling pathways differentially affect RNA polymerase II initiation, pausing, and elongation rate in cells. Mol Cell 50: 212-222. doi:10.1016/j.molcel.2013.02 .015

Davis CA, Ares M Jr. 2006. Accumulation of unstable promoterassociated transcripts upon loss of the nuclear exosome subunit Rrp6p in Saccharomyces cerevisiae. Proc Natl Acad Sci 103: 3262-3267. doi:10.1073/pnas.0507783103

de la Mata M, Alonso CR, Kadener S, Fededa JP, Blaustein M, Pelisch F, Cramer P, Bentley D, Kornblihtt AR. 2003. A slow RNA polymerase II affects alternative splicing in vivo. Mol Cell 12: 525-532. doi:10.1016/j.molcel.2003.08.001

Dobin A, Davis CA, Schlesinger F, Drenkow J, Zaleski C, Jha S, Batut P, Chaisson M, Gingeras TR. 2013. STAR: ultrafast universal RNA-seq aligner. Bioinformatics 29: 15-21. doi:10 $.1093 /$ bioinformatics/bts635

Estill M, Kerwin-Iosue CL, Wykoff DD. 2015. Dissection of the PHO pathway in Schizosaccharomyces pombe using epistasis and the alternate repressor adenine. Curr Genet 61: 175-183. doi:10.1007/s00294-014-0466-6

Exinger F, Lacroute F. 1992. 6-Azauracil inhibition of GTP biosynthesis in Saccharomyces cerevisiae. Curr Genet 22: 9-11. doi:10.1007/BF00351735

Fauquenoy S, Migeot V, Finet O, Yague-Sanz C, Khorosjutina O, Ekwall K, Hermand D. 2018. Repression of cell differentiation by a cis-acting lincRNA in fission yeast. Curr Biol 28: 383391.e3. doi:10.1016/j.cub.2017.12.048

Fernandez R, Berro J. 2016. Use of a fluoride channel as a new selection marker for fission yeast plasmids and application to fast genome editing with CRISPR/Cas9. Yeast 33: 549-557. doi:10.1002/yea.3178

Fong N, Kim H, Zhou Y, Ji X, Qiu J, Saldi T, Diener K, Jones K, Fu XD, Bentley DL. 2014. Pre-mRNA splicing is facilitated by an optimal RNA polymerase II elongation rate. Genes Dev 28: 2663-2676. doi:10.1101/gad.252106.114
Fong N, Brannan K, Erickson B, Kim H, Cortazar MA, Sheridan RM, Nguyen T, Karp S, Bentley DL. 2015. Effects of transcription elongation rate and Xrn2 exonuclease activity on RNA polymerase II termination suggest widespread kinetic competition. Mol Cell 60: 256-267. doi:10.1016/j.molcel.2015.09 .026

Garg A, Sanchez AM, Shuman S, Schwer B. 2018. A long noncoding (lnc)RNA governs expression of the phosphate transporter Pho84 in fission yeast and has cascading effects on the flanking prt lncRNA and pho1 genes. J Biol Chem 293: 4456-4467. doi:10.1074/jbc.RA117.001352

Gauthier S, Coulpier F, Jourdren L, Merle M, Beck S, Konrad M, Daignan-Fornier B, Pinson B. 2008. Co-regulation of yeast purine and phosphate pathways in response to adenylic nucleotide variations. Mol Microbiol 68: 1583-1594. doi:10.1111/j .1365-2958.2008.06261.x

Gómez-Herreros F, de Miguel-Jiménez L, Millán-Zambrano G, Peñate $\mathrm{X}$, Delgado-Ramos L, Muñoz-Centeno MC, Chávez S. 2012. One step back before moving forward: regulation of transcription elongation by arrest and backtracking. FEBS Lett 586: 2820-2825. doi:10.1016/j.febslet.2012.07.030

Harlen KM, Churchman LS. 2017. The code and beyond: transcription regulation by the RNA polymerase II carboxy-terminal domain. Nat Rev Mol Cell Biol 18: 263-273.

Hayashi A, Tanaka K. 2019. Short-homology-mediated CRISPR/ Cas9-based method for genome editing in fission yeast. G3 (Bethesda) 9: 1153-1163.

Hazelbaker DZ, Marquardt S, Wlotzka W, Buratowski S. 2013. Kinetic competition between RNA Polymerase II and Sen1-dependent transcription termination. Mol Cell 49: 55-66. doi:10.1016/j.molcel.2012.10.014

Hirota K, Miyoshi T, Kugou K, Hoffman CS, Shibata T, Ohta K. 2008. Stepwise chromatin remodelling by a cascade of transcription initiation of non-coding RNAs. Nature 456: 130 134. doi: $10.1038 /$ nature 07348

Jimeno-González S, Haaning LL, Malagon F, Jensen TH. 2010. The yeast $5^{\prime}-3^{\prime}$ exonuclease Ratlp functions during transcription elongation by RNA polymerase II. Mol Cell 37: 580-587. doi:10.1016/j.molcel.2010.01.019

Jonkers I, Kwak H, Lis JT. 2014. Genome-wide dynamics of Pol II elongation and its interplay with promoter proximal pausing, chromatin, and exons. Elife 3: e02407. doi:10.7554/eLife .02407

Kaikkonen MU, Adelman K. 2018. Emerging roles of non-coding RNA transcription. Trends Biochem Sci 43: 654-667. doi:10 .1016/j.tibs.2018.06.002

Kim M, Krogan NJ, Vasiljeva L, Rando OJ, Nedea E, Greenblatt JF, Buratowski S. 2004. The yeast Ratl exonuclease promotes transcription termination by RNA polymerase II. Nature 432: 517-522. doi:10.1038/nature03041

Kornblihtt AR, de la Mata M, Fededa JP, Munoz MJ, Nogues G. 2004. Multiple links between transcription and splicing. RNA 10: 1489-1498. doi:10.1261/rna.7100104

Kuehner JN, Brow DA. 2019. Regulation of a eukaryotic gene by GTP-dependent start site selection and transcription attenuation. Mol Cell 74: 634. doi:10.1016/j.molcel.2019.04.013

Larochelle M, Robert M-A, Hébert J-N, Liu X, Matteau D, Rodrigue S, Tian B, Jacques PE, Bachand F. 2018. Common mechanism of transcription termination at coding and noncoding RNA genes in fission yeast. Nat Commun 9: 4364. doi:10.1038/s41467-018-06546-x

Lemay JF, Larochelle M, Marguerat S, Atkinson S, Bähler J, Bachand F. 2014. The RNA exosome promotes transcription termination of backtracked RNA polymerase II. Nat Struct Mol Biol 21: 919-926. doi:10.1038/nsmb.2893 
Lemay JF, Marguerat S, Larochelle $M$, Liu X, van Nues R, Hunyadkürti J, Hoque M, Tian B, Granneman S, Bähler J, et al. 2016. The Nrd1-like protein Seb1 coordinates cotranscriptional $3^{\prime}$ end processing and polyadenylation site selection. Genes Dev 30: 1558-1572. doi:10.1101/gad .280222 .116

Li HD, Funk CC, Price ND. 2020. iREAD: a tool for intron retention detection from RNA-seq data. BMC Genomics 21: 128. doi:10.1186/s12864-020-6541-0

Liao Y, Smyth GK, Shi W. 2014. featureCounts: an efficient general purpose program for assigning sequence reads to genomic features. Bioinformatics 30: 923-930. doi:10.1093/bioinfor matics/btt656

Liu X, Freitas J, Zheng D, Oliveira MS, Hoque M, Martins T, Henriques T, Tian B, Moreira A. 2017a. Transcription elongation rate has a tissue-specific impact on alternative cleavage and polyadenylation in Drosophila melanogaster. RNA 23: 1807-1816. doi:10.1261/rna.062661.117

Liu X, Hoque M, Larochelle M, Lemay JF, Yurko N, Manley JL, Bachand F, Tian B. 2017b. Comparative analysis of alternative polyadenylation in $S$. cerevisiae and $S$. pombe. Genome Res 27: 1685-1695. doi:10.1101/gr.222331.117

Ljungdahl PO, Daignan-Fornier B. 2012. Regulation of amino acid, nucleotide, and phosphate metabolism in Saccharomyces cerevisiae. Genetics 190: 885-929. doi:10.1534/genetics .111 .133306

Love MI, Huber W, Anders S. 2014. Moderated estimation of fold change and dispersion for RNA-seq data with DESeq2. Genome Biol 15: 550. doi:10.1186/s13059-014-0550-8

Lunde BM, Reichow SL, Kim M, Suh H, Leeper TC, Yang F, Mutschler H, Buratowski S, Meinhart A, Varani G. 2010. Cooperative interaction of transcription termination factors with the RNA polymerase II C-terminal domain. Nat Struct Mol Biol 17: 1195-1201. doi:10.1038/nsmb.1893

Malagon F, Kireeva ML, Shafer BK, Lubkowska L, Kashlev M, Strathern JN. 2006. Mutations in the Saccharomyces cerevisiae RPB1 gene conferring hypersensitivity to 6-azauracil. Genetics 172: 2201-2209. doi:10.1534/genetics.105 .052415

Martens JA, Laprade L, Winston F. 2004. Intergenic transcription is required to repress the Saccharomyces cerevisiae SER3 gene. Nature 429: 571-574. doi:10.1038/nature02538

Martens JA, Wu PY, Winston F. 2005. Regulation of an intergenic transcript controls adjacent gene transcription in Saccharomyces cerevisiae. Genes Dev 19: 2695-2704. doi:10.1101/gad .1367605

Mason PB, Struhl K. 2005. Distinction and relationship between elongation rate and processivity of RNA polymerase II in vivo. Mol Cell 17: 831-840. doi:10.1016/j.molcel.2005.02.017

Mayr C. 2016. Evolution and biological roles of alternative 3'UTRs. Trends Cell Biol 26: 227-237. doi:10.1016/j.tcb.2015 .10 .012

Mayr C. 2017. Regulation by 3'-untranslated regions. Annu Rev Genet 51: 171-194. doi:10.1146/annurev-genet-120116024704

Meinhart A, Cramer P. 2004. Recognition of RNA polymerase II carboxy-terminal domain by $3^{\prime}$-RNA-processing factors. $\mathrm{Na}$ ture 430: 223-226. doi:10.1038/nature02679

Mercier A, Watt S, Bähler J, Labbé S. 2008. Key function for the CCAAT-binding factor Php4 to regulate gene expression in response to iron deficiency in fission yeast. Eukaryot Cell 7: 493-508. doi:10.1128/EC.00446-07

Parsa JY, Boudoukha S, Burke J, Homer C, Madhani HD. 2018. Polymerase pausing induced by sequence-specific RNA-binding protein drives heterochromatin assembly. Genes Dev 32: $953-$ 964. doi:10.1101/gad.310136.117

Pinto PA, Henriques T, Freitas MO, Martins T, Domingues RG, Wyrzykowska PS, Coelho PA, Carmo AM, Sunkel CE, Proudfoot NJ, et al. 2011. RNA polymerase II kinetics in polo polyadenylation signal selection. EMBO J 30: 2431-2444. doi:10 .1038/emboj.2011.156

Porrua O, Boudvillain M, Libri D. 2016. Transcription termination: variations on common themes. Trends in Genetics: TIG 32: 508-522. doi:10.1016/j.tig.2016.05.007

Proudfoot NJ. 2016. Transcriptional termination in mammals: stopping the RNA polymerase II juggernaut. Science 352: aad9926. doi:10.1126/science.aad9926

Reines D. 2003. Use of RNA yeast polymerase II mutants in studying transcription elongation. Meth Enzymol 371: 284292. doi:10.1016/S0076-6879(03)71021-0

Roberts GC, Gooding C, Mak HY, Proudfoot NJ, Smith CW. 1998. Co-transcriptional commitment to alternative splice site selection. Nucleic Acids Res 26: 5568-5572. doi:10.1093/nar/ 26.24.5568

Saldi T, Fong N, Bentley DL. 2018. Transcription elongation rate affects nascent histone pre-mRNA folding and $3^{\prime}$ end processing. Genes Dev 32: 297-308. doi:10.1101/gad.310896 .117

Sanchez AM, Shuman S, Schwer B. 2018a. Poly(A) site choice and Pol2 CTD Serine-5 status govern lncRNA control of phosphate-responsive tgp1 gene expression in fission yeast. $R N A$ 24: 237-250. doi:10.1261/rna.063966.117

Sanchez AM, Shuman S, Schwer B. 2018b. RNA polymerase II CTD interactome with $3^{\prime}$ processing and termination factors in fission yeast and its impact on phosphate homeostasis. Proc Natl Acad Sci 115: E10652-E10661. doi:10.1073/pnas .1810711115

Schwer B, Sanchez AM, Garg A, Chatterjee D, Shuman S. 2017. Defining the DNA binding site recognized by the fission yeast $\mathrm{Zn}_{2} \mathrm{Cys}_{6}$ transcription factor Pho7 and its role in phosphate homeostasis. MBio 8: e01218-17. doi:10.1128/mBio .01218-17

Shah S, Wittmann S, Kilchert C, Vasiljeva L. 2014. IncRNA recruits RNAi and the exosome to dynamically regulate phol expression in response to phosphate levels in fission yeast. Genes Dev 28: 231-244. doi:10.1101/gad.230177.113

Shaw RJ, Reines D. 2000. Saccharomyces cerevisiae transcription elongation mutants are defective in PUR5 induction in response to nucleotide depletion. Mol Cell Biol 20: 7427-7437. doi:10.1128/MCB.20.20.7427-7437.2000

Sheridan RM, Fong N, D'Alessandro A, Bentley DL. 2019. Widespread backtracking by RNA Pol II is a major effector of gene activation, 5' pause release, termination, and transcription elongation rate. Mol Cell 73: 107-118.e4. doi:10.1016/j .molcel.2018.10.031

Strathern J, Malagon F, Irvin J, Gotte D, Shafer B, Kireeva M, Lubkowska L, Jin DJ, Kashlev M. 2013. The fidelity of transcription: RPB1 (RPO21) mutations that increase transcriptional slippage in S. cerevisiae. J Biol Chem 288: 2689-2699. doi:10 $.1074 /$ ibc.M112.429506

Sun M, Schwalb B, Schulz D, Pirkl N, Etzold S, Lariviere L, Maier KC, Seizl M, Tresch A, Cramer P. 2012. Comparative dynamic transcriptome analysis (cDTA) reveals mutual feedback between mRNA synthesis and degradation. Genome Res 22: 1350-1359. doi:10.1101/gr.130161.111

Thiebaut M, Colin J, Neil H, Jacquier A, Séraphin B, Lacroute F, Libri D. 2008. Futile cycle of transcription initiation and termination modulates the response to nucleotide shortage in 
S. cerevisiae. Mol Cell 31: 671-682. doi:10.1016/j.molcel.2008 .08 .010

Tian B, Manley JL. 2017. Alternative polyadenylation of mRNA precursors. Nat Rev Mol Cell Biol 18: 18-30. doi:10.1038/ nrm.2016.116

Touat-Todeschini L, Shichino Y, Dangin M, Thierry-Mieg N, Gilquin B, Hiriart E, Sachidanandam R, Lambert E, Brettschneider J, Reuter $M$, et al. 2017. Selective termination of lncRNA transcription promotes heterochromatin silencing and cell differentiation. EMBO $J$ 36: 2626-2641. doi:10 $.15252 / \mathrm{embj} .201796571$

Veloso A, Kirkconnell KS, Magnuson B, Biewen B, Paulsen MT, Wilson TE, Ljungman M. 2014. Rate of elongation by RNA polymerase II is associated with specific gene features and epigenetic modifications. Genome Res 24: 896-905. doi:10.1101/gr .171405 .113
West S, Gromak N, Proudfoot NJ. 2004. Human 5' $\rightarrow 3^{\prime}$ exonuclease Xrn2 promotes transcription termination at co-transcriptional cleavage sites. Nature 432: 522-525. doi:10.1038/ nature 03035

Wickiser JK, Winkler WC, Breaker RR, Crothers DM. 2005. The speed of RNA transcription and metabolite binding kinetics operate an FMN riboswitch. Mol Cell 18: 49-60. doi:10 .1016/j.molcel.2005.02.032

Wittmann S, Renner M, Watts BR, Adams O, Huseyin M, Baejen C, El Omari K, Kilchert C, Heo DH, Kecman T, et al. 2017. The conserved protein Sebl drives transcription termination by binding RNA polymerase II and nascent RNA. Nat Commun 8: 14861. doi:10.1038/ncomms14861

Zaborowska J, Egloff S, Murphy S. 2016. The pol II CTD: new twists in the tail. Nat Struct Mol Biol 23: 771-777. doi:10 $.1038 / \mathrm{nsmb} .3285$ 


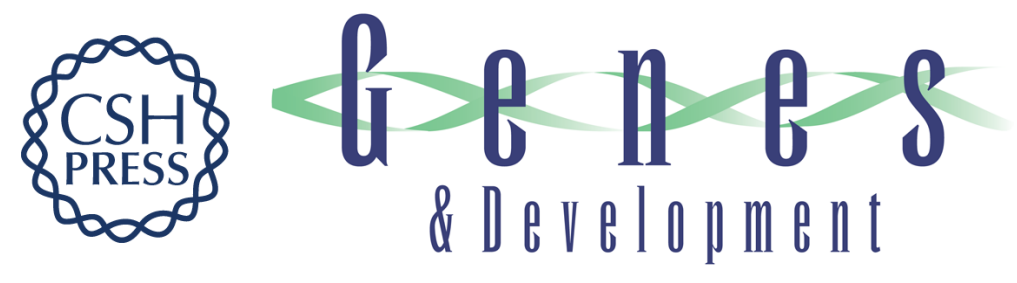

\section{Nutrient-dependent control of RNA polymerase II elongation rate regulates specific gene expression programs by alternative polyadenylation}

Carlo Yague-Sanz, Yann Vanrobaeys, Ronan Fernandez, et al.

Genes Dev. 2020, 34: originally published online June 4, 2020

Access the most recent version at doi:10.1101/gad.337212.120

Supplemental http://genesdev.cshlp.org/content/suppl/2020/06/03/gad.337212.120.DC1

Material

References This article cites 81 articles, 27 of which can be accessed free at: http://genesdev.cshlp.org/content/34/13-14/883.full.html\#ref-list-1

Creative This article, published in Genes \& Development, is available under a Creative Commons

Commons License (Attribution 4.0 International), as described at

License http://creativecommons.org/licenses/by/4.0/.

Email Alerting Receive free email alerts when new articles cite this article - sign up in the box at the top Service right corner of the article or click here.

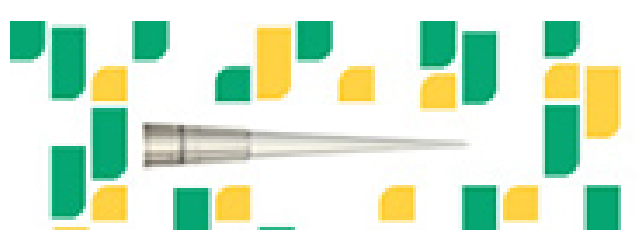

Focused on your science. 